\title{
Multi-frequency VLA observations of a new sample of CSS/GPS radio sources
}

\author{
C. Fanti ${ }^{1,2}$, F. Pozzi ${ }^{1,3}$, D. Dallacasa ${ }^{2,3}$, R. Fanti ${ }^{1,2}$, L. Gregorini $^{1,2}$, C. Stanghellini ${ }^{4}$, and M. Vigotti ${ }^{2}$ \\ 1 Dipartimento di Fisica, Università di Bologna, Via Irnerio 46, 40126 Bologna, Italy \\ 2 Dipartimento di Astronomia, Università di Bologna, Via Ranzani 1, 40127 Bologna, Italy \\ 3 Istituto di Radioastronomia del CNR, Via Gobetti 101, 40129 Bologna, Italy \\ 4 Istituto di Radioastronomia del CNR, CP 141, 96017 Noto SR, Italy
}

Received 12 October 2000 / Accepted 3 January 2001

\begin{abstract}
In this paper we present a new sample of 87 Compact Steep Spectrum radio sources (CSS) with flux density $\geq 0.8 \mathrm{Jy}$ at $0.4 \mathrm{GHz}$. This sample has been selected from the B3-VLA sample with the aid of new VLA observations at 4.9 and $8.5 \mathrm{GHz}$ which allowed to clean an earlier selection based on VLA $1.5 \mathrm{GHz}$ data. Redshifts, either spectroscopic or photometric, are known for $62 \%$ of the sources. About $75 \%$ of the sources are resolved or slightly resolved in the new observations. The range of measured linear sizes is from $20 h^{-1} \mathrm{kpc}$ (selection upper limit) down to $\approx 0.5 \mathrm{~h}^{-1} \mathrm{kpc}$, which corresponds to our resolution limit. The Largest Linear Size $(L L S)$ distribution is well represented by the power law $\mathrm{d} N / \mathrm{d}(L L S) \propto L L S^{-0.6}$, consistent with earlier results. The majority of the radio sources smaller than $0.5 h^{-1} \mathrm{kpc}$ shows a marked spectral flattening at low frequencies. The four frequency spectra computed in the range $0.4-8.5 \mathrm{GHz}$ display a significant steepening at high frequencies in the largest majority of cases. A considerable fraction of source components are polarized, with median values of their fractional polarization $\approx 6 \%$ and $\approx 4 \%$ at 8.5 and $4.9 \mathrm{GHz}$ respectively.
\end{abstract}

Key words. catalogs - galaxies: active - radio continuum: galaxies - quasar: general - polarization - radio spectra

\section{Introduction}

Compact Steep Spectrum (CSS) and GHz Peaked Spectrum (GPS) radio sources are extragalactic objects mostly as powerful as the classical FRII radio sources, but of much smaller physical size (Largest Linear Size, $L L S$, $\lesssim 20 h^{-1} \mathrm{kpc}^{1}$ ). Yet they have a normal/steep radio spectrum at $\mathrm{GHz}$ frequencies, i.e. they are not core-dominated. In several of them the radio spectrum shows a flattening or a marked turnover (usually attributed to synchrotron self-absorption) at frequencies between $\approx 100 \mathrm{MHz}$ and a few GHz. Those with a turnover frequency $\geq 1 \mathrm{GHz}$ are the GPS sources. A comprehensive review on the subject is presented by O'Dea (1998).

It is mostly accepted that, at least the two-sided (or symmetric) objects of this class, represent the young stage in the radio source evolution, from Compact through Medium to Large Symmetric Objects (CSO, MSO, LSO,

\footnotetext{
Send offprint requests to: D. Dallacasa,

e-mail: ddallaca@ira.bo.cnr.it

${ }^{1} h=H_{0} / 100, q_{0}=0.5$.
}

defined as in Fanti et al. 1995; Readhead et al. 1996). Indeed in a handful of CSOs expansion velocities of up to $\approx 0.4 c$ have been measured (see, e.g., Owsianik \& Conway 1998; Owsianik et al. 1998; Polatidis et al. 1999; Taylor et al. 2000; Tschager et al. 2000) proving that at least these radio sources were born not earlier than a few thousand years ago. For MSOs no such direct measures exist, however a statistical study by Murgia et al. (1999) on the high frequency steepening of their radio spectrum proves that, if this is due to electron radiative ageing, such objects are likely to be younger than about $10^{5}$ years.

Phenomenological models, based on the numberdiameter counts, developed independently by Fanti et al. (1995) and Readhead et al. (1996) show that, in the scenario in which these objects are young (youth scenario, see Fanti 2000 for a short review) the high proportion (15-30\%) of CSS/GPS in radio catalogues requires an evolution of the radio source luminosity approximately as the $L L S^{-0.5}$. This is consistent with the continuum energy supply radio source models (Baldwin 1982; Begelman 1996; Kaiser et al. 1997; Blundell et al. 1999). The youth 
model is based on relatively small numbers $(\approx 40)$ of high power objects, taken from the $3 C R$ (Laing et al. 1983), the $P W$ (Peacock \& Wall 1982) (the $3 C R \& P W$ sample) and the $P R$ (Pearson \& Readhead 1988) samples with sizes ranging from few hundreds of pc up to $\approx 20 h^{-1} \mathrm{kpc}$. Recently Stanghellini et al. (1998), Snellen et al. (1998) and Marecki et al. (1999) have selected samples of GPSs which allow to probe the size distribution down to $\approx 10 h^{-1}$ pc and to partially test the models.

In order to constrain the models it is important to extend the statistics in number of objects, size range and radio power. We have therefore selected a new complete sample of 87 CSSs from the B3-VLA catalogue (Sect. 2). The sample is presented in this paper and, together with VLBI observations presently being analysed (Sect. 7), it will allow to determine the $L L S$ distribution from $\approx 100 h^{-1} \mathrm{pc}$ up to $\approx 20 h^{-1} \mathrm{kpc}$, with a statistics twice as good than from the CSS samples studied so far. In this paper, the first of a series, we present and briefly discuss the VLA observations. Forthcoming papers will present the VLBI observations and discuss more deeply the properties of the sources and their relation with the models.

The layout of the paper is the following:

- Section 2 recalls the relevant points of the B3-VLA catalogue;

- Section 3 defines the new sample of CSSs selected from the aforementioned catalogue;

- Section 4 describes the new VLA observations and data reduction;

- Observational results are presented in Sect. 5;

- A concise discussion is given in Sect. 6;

- A short summary of this work along with future plans can be found in Sect. 7 .

\section{The B3-VLA sample}

The B3-VLA sample (Vigotti et al. 1989) consists of 1049 radio sources selected from the $408 \mathrm{MHz}$ B3 catalogue (Ficarra et al. 1985). They are grouped into 5 different flux density bins $(0.1-0.2,0.2-0.4,0.4-0.8,0.8-1.6$ and $>1.6 \mathrm{Jy})$ and cover the R.A. intervals $23^{\mathrm{h}} 00^{\mathrm{m}}-03^{\mathrm{h}} 00^{\mathrm{m}}$ and $07^{\mathrm{h}} 00^{\mathrm{m}}-15^{\mathrm{h}} 00^{\mathrm{m}}$ within the declination range of the B3 catalogue $\left(37^{\circ} 15^{\prime}-47^{\circ} 37^{\prime}\right)$, in strips of different widths in such a way that each flux density bin counts approximately the same number of objects $(\approx 200-250)$. All these sources have been observed between November 1981 and March 1983 with the VLA at $1.465 \mathrm{GHz}$ in $\mathrm{C}$ and A configurations (hereafter $V L A-C$ and $V L A-A$ ), with resolutions of $\approx 14$ and 1.4 arcsec. Vigotti et al. (1999) presented a spectral database with flux densities at $151,327,408 \mathrm{MHz}$ and $1.4,4.85$ and $10.6 \mathrm{GHz}$, for the whole sample.

A systematic program of optical identifications, based on the $V L A-A$ radio positions, was carried out by Vigotti et al. (1989) using the Palomar Observatory Sky Survey $(P O S S)$ prints. A long term program is presently being carried out to obtain the spectroscopic redshifts for the whole B3-VLA sample (Thompson et al. 1994; Thompson et al. in prep.). The status of this program at present is as follows:

a) all quasar candidates visible on the POSS prints have been observed spectroscopically and their redshifts are available (Vigotti et al. 1990);

b) for galaxies visible on the POSS prints $\left(m_{R} \lesssim 20.5\right)$ " $R$-photometric" redshifts (up to $z \approx 0.5$ ) have been determined, with an accuracy of $\approx 20 \%$, following the method described in Vigotti et al. (1989); only a minority of them have also spectroscopic redshifts;

c) deeper optical identifications, in $R$ and/or $K$ band, have produced, so far, new identifications with fainter galaxies and a very few quasars for $\approx 40 \%$ of the sources unidentified on the POSS prints. The $K$ magnitudes have been used to estimate " $K$-photometric" redshifts from the redshift-magnitude relation (Thompson et al. in prep.);

d) for the sources in point c) a number of spectroscopic redshifts were also obtained.

Radio sources without an identification (optically fainter than $m_{R} \approx 20$ ) are referred to as "Empty Fields" $(E)$.

\section{The B3-VLA sample of CSS candidates}

The sample discussed in this paper is based on $1.5 \mathrm{GHz}$ $V L A-A$ data (Vigotti et al. in prep.). We recall briefly the relevant details of those observations and data reduction procedure.

Each source was observed with a single snap-shot of about $2 \mathrm{~min}$. The data reduction was performed in a standardized way, using a sort of pipe-line, and images of $512 \times 512$ pixels 0.5 arcsec in size, were produced. In most cases this procedure gave good-quality (for the epoch) images with an $\mathrm{rms}$ noise of $\sim 0.3 \mathrm{mJy}$. The calibration uncertainties were estimated to be about $5 \%$. We have rereduced the data for those CSS candidates for which the outcome of the pipe-line was not satisfactorily enough. From these images (resolution $\approx 1.4$ arcsec), we selected those sources with projected linear sizes $\lesssim 20 h^{-1} \mathrm{kpc}$ and $\alpha \geq 0.5\left(S \propto \nu^{-\alpha}\right)$ above $1.5 \mathrm{GHz}$ (usually up to $10 \mathrm{GHz}$ ). Typically this linear size cut corresponds to a cut in the Largest Angular Size $(L A S) \lesssim 5$ arcsec, except for 3 nearby radio galaxies with sizes $\gtrsim 6$ arcsec. Because of the modest angular resolution, some uncertainty may arise in the selection at the upper boundary of the linear sizes. We estimate, however, that no more than one or two sources have been missed because erroneously considered too large. In order to match the Largest Linear Size upper limit of the selection, for the radio sources without redshift we adopted $z=1.05$, which is the median redshift of the sources originally unidentified on the POSS prints and for which photometric or spectroscopic redshifts have become subsequently available (points c) and d) of Sect. 2). This value is close to $z=1.25$ which, for a given angular size, maximizes the linear size for $q_{0}=0.5$. Such linear sizes are therefore upper limits and, given their dependence on $z$, they are correct to within $5 \%$ for $0.8 \lesssim z \lesssim 2.1$. 
We estimate that only one or two of the nearest objects (but still at $z>0.5$, see Sect. 2) might have been lost at the $20 h^{-1} \mathrm{kpc}$ boundary. For the radio power of the objects without redshift (Tables 1 and 2) we computed instead a lower limit, corresponding to $z=0.5$.

The B3-VLA catalogue contains a total of $\approx 275$ such CSS/GPS candidates. Since the number of existing redshifts, either spectroscopic or photometric, is satisfactorily high $(>60 \%)$ only in the highest flux density bins $\left(S_{0.4} \geq 0.8 \mathrm{Jy}\right)$ and this number is expected to increase further in the near future, we concentrated our selection on this "bright" sub-sample. We obtained 98 objects. Only $\approx 32 \%$ of them were well resolved at the 1.4 arcsec resolution of the $V L A-A$ at $1.5 \mathrm{GHz}$. In order to obtain structure information at higher resolutions we have re-observed all of them with the $V L A-A$ at 4.9 and $8.5 \mathrm{GHz}$.

\section{The VLA 4.9 and $8.5 \mathrm{GHz}$ observations and data reduction}

Observations were made on February 22nd 1998, with the $V L A-A$ virtually simultaneously at 4.86 and $8.46 \mathrm{GHz}$, with two $50 \mathrm{MHz}$ IFs at each frequency. This provides a resolution of about 0.4 and 0.2 arcsec respectively. The three sources with angular size larger than $6^{\prime \prime}$ and at a redshift $\lesssim 0.2$ were not observed. Since the sources are relatively strong and were expected to have small angular sizes, snap-shot observing mode was considered adequate. Each source was observed in a single snap-shot for a total integration time of 1 to $3.2 \mathrm{~min}$ at $8.5 \mathrm{GHz}$ and 1 to $2.5 \mathrm{~min}$ at $4.9 \mathrm{GHz}$, depending on the source flux density and structure estimated from the $1.5 \mathrm{GHz} V L A-A$ data.

Target sources have been interleaved with unresolved phase and amplitude calibration sources, observed about three times per hour, for 2 min. As primary flux density calibrator we used $3 \mathrm{C} 286$, following the NRAO AIPS COOKBOOK recipes for the various baselines. Calibration uncertainties are estimated to be about $3 \%$ at both frequencies. Suitable calibrators $(0742+103$ and $0108+388)$ were also observed at different parallactic angles to determine the instrumental polarization. The polarization position angle was calibrated with 3C138 and 3C48, considering again the instrument response at the various baselines. The residual instrumental polarization is evaluated to be $\lesssim 0.3 \%$ (rms). Most of the sources were observed close to meridian and thus with a circular beam. However, due to scheduling constraints, a number of objects were observed at the edges of the observing window and therefore have more elliptical beams. Extreme values reach $\approx 1.0^{\prime \prime} \times 0.4^{\prime \prime}$ and $\approx 0.5^{\prime \prime} \times 0.3^{\prime \prime}$ at 4.9 and $8.5 \mathrm{GHz}$ respectively. Beam sizes are shown, for each source, on the images in Figs. 3 and 5.

The whole data reduction has been performed in AIPS. After the standard phase and amplitude calibration for both total intensity and polarization, images have been produced by means of the task IMAGR. Two or three passes of phase self-calibration were generally required to obtain the final maps. Only in a minority of cases amplitude self-calibration was also necessary, and it was applied after phases had been satisfactorily corrected. The final noise $(1 \sigma)$ is usually in the range $\sim 50-80 \mu \mathrm{Jy} /$ beam and $40-60 \mu \mathrm{Jy} /$ beam at 4.9 and $8.5 \mathrm{GHz}$ respectively, in agreement with the expectations. Higher noise is found for sources observed out of meridian or, occasionally, for some moderate malfunction of the instrument. The dynamic range is estimated to be better than 2000:1 in most cases, and does not affect the noise appreciably in the largest majority of cases. The actual noise $(3 \sigma)$ is reported for each image in Figs. 3 and 5 as the value of the first contour (indicated in the figure as f.c.).

The new high frequency observations turned out to be more sensitive than old ones at $1.5 \mathrm{GHz}$, mostly because of the much improved instrumental and data reduction capabilities. Because of this, nine of the originally selected sources resulted to be more extended than believed, due to the presence of low brightness components not reliably detected earlier. Furthermore two sources $(0739+397 \mathrm{~B}$ and $0945+408$ ) were found to have a flatter radio spectrum in the whole explored frequency range and, therefore, were removed from the sample as well. Finally in five fields a weak source $\left(S_{1.5} \gtrsim 5 \mathrm{mJy}\right)$ is present, at a distance $\lesssim 30$ arcsec from the object listed in Table 1 (see Sect. 5.6). Considering the source counts at $1.412 \mathrm{MHz}$ by KatgertMerkelijn et al. (1985) we have estimated that, statistically, a couple of weak radio sources may fall by chance within $30^{\prime \prime}$ of our target object.

The final sample counts 87 CSSs $\left(L L S \lesssim 20 h^{-1} \mathrm{kpc}\right)$. They represent the $23 \%$ of the 374 radio sources in the flux density bins $S_{0.4} \geq 0.8 \mathrm{Jy}$, a proportion similar to that reported for the $3 C R \& P W$ sample by Fanti et al. (1995). The sample is given in Table 1, with the relevant information. Table 2 lists the eleven sources which have been excluded from the original selection.

The content of both Tables is as follows.

- Column 1: source name;

- Column 2: optical identification (Id); Q quasar ${ }^{2}, \mathbf{G}$ galaxy on the POSS prints, $\mathbf{E}$ no identification on the POSS prints, g galaxy fainter than the POSS limit;

Column 3: red magnitude; for the objects brighter than the POSS limit $\left(m_{R} \lesssim 20.5\right)$ they are taken from: i) the APM catalogue (http://www.ast.cam.ac.uk./ apmcat) for quasars; ii) the APS catalogue (http://aps.umn.edu) for galaxies. The others are new measurements (see Sect. 2);

- Column 4: redshift; $K$ and $R$ indicate a photometric redshift from $K$ or $R$ (from POSS prints) magnitude;

- Column 5: $408 \mathrm{MHz}$ flux density, in Jy;

- Column 6: Largest Angular Size (in arcsec) measured, in most cases, as separation between the outermost component peaks. A " " means measured on the image contour plot (see Sect. 5.1);

- Columns 7, 8: spectral indices $\alpha_{0.4}^{1.4}$ and $\alpha_{4.9}^{8.5}$. Note that we used the NVSS (Condon et al. 1998) flux densities at $1.4 \mathrm{GHz}$ instead of our own at $1.5 \mathrm{GHz}$ (Sect. 6.2);

\footnotetext{
${ }^{2}$ see note in Sect. 5.6 on the change of Id for $0951+422$.
} 
Table 1. The B3-VLA sample of CSS

\begin{tabular}{|c|c|c|c|c|c|c|c|c|c|c|c|}
\hline Name & Id & $m_{R}$ & $z$ & $\begin{array}{l}S_{0.4} \\
(\mathrm{Jy})\end{array}$ & $\begin{array}{r}L A S \\
(\operatorname{arcsec})\end{array}$ & $\alpha_{0.4}^{1.4}$ & $\alpha_{4.9}^{8.5}$ & $\begin{array}{r}\log P_{0.4} \\
\left(\mathrm{~W} / \mathrm{Hz} h^{-2}\right)\end{array}$ & $\begin{array}{r}L L S \\
\left(\operatorname{kpc} h^{-1}\right)\end{array}$ & \multicolumn{2}{|c|}{ Morph. } \\
\hline $0003+387$ & $\mathrm{~g}$ & 23.7 & 1.47 & 1.36 & 1.3 & 0.83 & 1.49 & 27.65 & 5.5 & $\mathrm{D}$ & \\
\hline $0034+444$ & $\mathrm{~g}$ & 22.8 & 2.79 & 2.25 & 3.2 & 0.95 & 1.27 & 28.53 & 11.9 & $\mathrm{scJ}$ & $\ddagger$ \\
\hline $0039+373$ & $\mathrm{~g}$ & & 1.006 & 2.00 & 0.1 & 0.59 & 1.40 & 27.37 & 0.5 & $\mathrm{U}$ & \\
\hline $0039+391$ & $\mathrm{~g}$ & & 1.01 & 1.02 & 0.34 & 0.96 & 1.38 & 27.19 & 1.5 & $\mathrm{SR}$ & \\
\hline $0039+398$ & $\mathrm{E}$ & & & 1.98 & 3.7 & 0.77 & 1.19 & $>26.8$ & {$[16]$} & D & $\ddagger$ \\
\hline $0039+412$ & $\mathrm{E}$ & & & 0.91 & 2.0 & 0.72 & 0.99 & $>26.4$ & {$[8.5]$} & $\mathrm{T}$ & \\
\hline $0041+425$ & $\mathrm{E}$ & & & 1.26 & 1.0 & 0.79 & 1.16 & $>26.6$ & {$[4.1]$} & $\mathrm{D}$ & $\ddagger$ \\
\hline $0049+379$ & $\mathrm{~g}$ & & $1.7 \mathrm{~K}$ & 2.55 & 1.45 & 0.92 & 1.58 & 28.10 & 6.1 & D & $\ddagger$ \\
\hline $0110+401$ & $\mathrm{Q}$ & 20.0 & 1.479 & 1.08 & $\sim 3.9$ & 0.55 & 0.71 & 27.44 & $\sim 16.7$ & $\mathrm{Cx}$ & \\
\hline $0120+405$ & $\mathrm{~g}$ & & 0.84 & 1.68 & 2.4 & 0.79 & 1.19 & 27.20 & 10.1 & $\mathrm{D}$ & $\ddagger$ \\
\hline $0123+402$ & $\mathrm{~g}$ & 23.8 & & 0.81 & 1.1 & 0.93 & 1.21 & $>26.4$ & {$[4.7]$} & $\mathrm{scJ} ?$ & $\ddagger$ \\
\hline $0128+394$ & $\mathrm{~g}$ & 22.9 & $1.6 \mathrm{~K}$ & 0.92 & 2.7 & 0.84 & 1.23 & 27.53 & 11.5 & $\mathrm{D}$ & $\ddagger$ \\
\hline $0137+401$ & $\mathrm{Q}$ & 18.5 & 1.62 & 0.82 & 4.2 & 0.84 & 1.03 & 27.48 & 17.6 & $\mathrm{~T}$ & $\ddagger$ \\
\hline $0140+387$ & $\mathrm{~g}$ & & $2.9 \mathrm{~K}$ & 1.81 & 0.7 & 1.17 & 1.71 & 28.55 & 2.6 & $\mathrm{D} ?$ & $\ddagger$ \\
\hline $0144+432$ & Q & 21.0 & 1.26 & 0.82 & $\sim 4.0$ & 0.71 & 1.18 & 27.21 & $\sim 17.2$ & $\mathrm{D}$ & $\ddagger$ \\
\hline $0147+400$ & $\mathrm{E}$ & & & 1.54 & 0.1 & 0.61 & 0.73 & $>26.6$ & {$[0.4]$} & $\mathrm{U}$ & \\
\hline $0213+412$ & $\mathrm{~g}$ & 21.4 & 0.515 & 1.26 & 2.0 & 0.66 & 0.80 & 26.58 & 7.3 & $\mathrm{~T}$ & $\ddagger$ \\
\hline $0222+422 \mathrm{~A}$ & $\mathrm{~g}$ & & $3.5 \mathrm{~K}$ & 0.81 & 3.4 & 0.92 & 1.39 & 28.24 & 11.9 & D & $\ddagger$ \\
\hline $0228+409 \mathrm{~A}$ & $\mathrm{E}$ & & & 0.90 & 3.8 & 0.74 & 1.10 & $>26.4$ & {$[16.2]$} & D & $\ddagger$ \\
\hline $0254+406$ & $\mathrm{~g}$ & 24.0 & 1.224 & 1.43 & $\sim 4.5$ & 0.84 & 1.10 & 27.49 & $\sim 18.9$ & $\mathrm{D}$ & $\ddagger$ \\
\hline $0255+460$ & $\mathrm{Q}$ & 20.5 & 1.21 & 1.74 & 0.66 & 0.71 & 1.10 & 27.51 & 2.8 & $\mathrm{D}$ & \\
\hline $0701+392$ & $\mathrm{Q}$ & 18.7 & 1.238 & 1.17 & 1.8 & 0.67 & 0.97 & 27.35 & 7.8 & $\mathrm{D}$ & $\ddagger$ \\
\hline $0703+468$ & $\mathrm{E}$ & & & 1.90 & 0.08 & 0.14 & 0.82 & $>26.6$ & {$[0.3]$} & $\mathrm{U}$ & \\
\hline $0722+393 \mathrm{~A}$ & $\mathrm{E}$ & & & 2.91 & 0.25 & 0.77 & 1.26 & $>26.9$ & {$[1.0]$} & $\mathrm{SR}$ & $\ddagger$ \\
\hline $0729+437$ & $\mathrm{E}$ & & & 0.90 & 1.3 & 0.68 & 0.91 & $>26.4$ & {$[5.6]$} & $\mathrm{D}$ & $\ddagger$ \\
\hline $0744+464$ & $\mathrm{~g}$ & & 2.926 & 1.66 & 1.4 & 0.95 & 1.05 & 28.46 & 5.1 & $\mathrm{~T}$ & $\ddagger$ \\
\hline $0748+413 B$ & $\mathrm{E}$ & & & 0.83 & $\sim 0.4$ & 1.02 & 1.07 & $>26.4$ & {$[\sim 1.8]$} & SR & $\ddagger$ \\
\hline $0754+396$ & $\mathrm{~g}$ & & 2.119 & 1.53 & $\sim 2.2$ & 0.82 & 1.17 & 28.06 & $\sim 8.9$ & scJ & $\ddagger$ \\
\hline $0800+472$ & $\mathrm{E}$ & & & 2.06 & $\sim 1.0$ & 0.66 & 0.73 & $>26.8$ & {$[\sim 4.3]$} & $\mathrm{scJ} ?$ & $\ddagger$ \\
\hline $0805+406$ & $\mathrm{E}$ & & & 1.33 & $\sim 2.5$ & 0.74 & 0.97 & $>26.6$ & {$[\sim 10.7]$} & $\mathrm{T} ?$ & $\ddagger$ \\
\hline $0809+404$ & $\mathrm{~g}$ & & 0.551 & 2.31 & 1.2 & 0.62 & 0.80 & 26.9 & 4.5 & $\mathrm{Da}$ & $\ddagger$ \\
\hline $0810+460 \mathrm{~B}$ & G & 20.3 & $0.33 \mathrm{R}$ & 4.08 & 0.63 & 1.05 & 1.20 & 26.74 & 1.8 & D & $\ddagger$ \\
\hline $0814+441$ & $\mathrm{E}$ & & & 0.84 & 4.0 & 0.88 & 0.93 & $>26.4$ & {$[17.0]$} & Dc & $\ddagger$ \\
\hline $0822+394$ & $\mathrm{~g}$ & & 1.18 & 1.76 & 0.05 & 0.30 & 1.02 & 27.33 & 0.2 & $\mathrm{U}$ & \\
\hline $0840+424 \mathrm{~A}$ & $\mathrm{E}$ & & & 2.28 & 0.08 & 0.39 & 0.76 & $>26.8$ & {$[0.3]$} & $\mathrm{U}$ & \\
\hline $0856+406$ & $\mathrm{~g}$ & 24.3 & 2.28 & 1.07 & 0.8 & 1.22 & 1.79 & 28.10 & 3.2 & $\mathrm{D}$ & $\ddagger$ \\
\hline $0902+416$ & $\mathrm{E}$ & & & 0.93 & 0.34 & 0.50 & 0.75 & $>26.4$ & {$[1.4]$} & SR & \\
\hline $0930+389$ & $\mathrm{~g}$ & & 2.395 & 0.92 & 3.7 & 0.94 & 1.05 & 27.97 & 14.4 & Dc & $\ddagger$ \\
\hline $0935+428 \mathrm{~A}$ & $\mathrm{~g}$ & 24.0 & 1.291 & 1.35 & 1.3 & 0.90 & 1.09 & 27.54 & 5.5 & $\mathrm{D}$ & \\
\hline $0951+422$ & $\mathrm{Q} \ddagger$ & 20.5 & 1.783 & 1.08 & 2.0 & 0.74 & 0.77 & 27.64 & 8.3 & $\mathrm{~T}$ & $\ddagger$ \\
\hline $0955+390$ & $\mathrm{E}$ & & & 1.05 & $\sim 4.8$ & 0.65 & 0.79 & $>26.5$ & {$[\sim 19.2]$} & Dc & $\ddagger$ \\
\hline $1007+422$ & $\mathrm{E}$ & & & 1.00 & 0.13 & 0.67 & 0.90 & $>26.4$ & {$[0.55]$} & $\mathrm{U}$ & \\
\hline $1008+423$ & $\mathrm{E}$ & & & 1.12 & 0.05 & 0.51 & 0.78 & $>26.5$ & {$[0.2]$} & $\mathrm{U}$ & \\
\hline $1014+392$ & G & 19.1 & 0.206 & 3.08 & 6.1 & 0.63 & & 26.16 & 13.2 & $\mathrm{Da}$ & \\
\hline $1016+443$ & G & 19.7 & $0.33 \mathrm{R}$ & 1.00 & 0.11 & 0.83 & 1.29 & 26.11 & 0.3 & $\mathrm{U}$ & \\
\hline $1025+390 \mathrm{~B}$ & G & 18.5 & 0.361 & 1.54 & $\sim 3.2$ & 0.67 & 0.54 & 26.36 & $\sim 9.7$ & Dc & $\ddagger$ \\
\hline $1027+392$ & $\mathrm{E}$ & & & 0.83 & $\sim 1.6$ & 0.61 & 0.75 & $>26.4$ & {$[\sim 6.9]$} & $\mathrm{scJ}$ & $\ddagger$ \\
\hline $1039+424$ & $\mathrm{E}$ & & & 0.87 & $\sim 1.5$ & 0.91 & 1.31 & $>26.4$ & {$[\sim 6.5]$} & $\mathrm{scJ}$ & $\ddagger$ \\
\hline $1044+454$ & $\mathrm{~g}$ & 24.8 & $4.1 \mathrm{~K}$ & 1.67 & 1.0 & 1.08 & 1.43 & 28.82 & 3.2 & $\mathrm{Da}$ & $\ddagger$ \\
\hline $1049+384$ & $\mathrm{~g}$ & 20.9 & 1.018 & 1.24 & 0.1 & 0.47 & 1.17 & 27.14 & 0.4 & $\mathrm{U}$ & \\
\hline $1055+404 \mathrm{~A}$ & $\mathrm{E}$ & & & 0.94 & 2.8 & 0.76 & 0.99 & $>26.5$ & [12.1] & fcJ & $\ddagger$ \\
\hline $1128+455$ & $\mathrm{G}$ & 18.7 & 0.40 & 4.37 & $\sim 0.9$ & 0.62 & 0.90 & 26.98 & $\sim 2.9$ & $\mathrm{scJ}$ & \\
\hline $1133+432$ & $\mathrm{E}$ & & & 0.88 & 0.07 & -0.41 & 1.00 & $>26.3$ & {$[0.3]$} & $\mathrm{U}$ & $\ddagger$ \\
\hline $1136+383$ & $\mathrm{E}$ & & & 0.84 & 0.05 & 0.47 & 0.75 & $>26.4$ & {$[0.2]$} & $\mathrm{U}$ & \\
\hline $1136+420$ & $\mathrm{~g}$ & 21.7 & 0.829 & 1.09 & 1.0 & 0.67 & 0.84 & $>26.5$ & 4.3 & $\mathrm{Da}$ & \\
\hline $1141+466$ & G & 15.5 & 0.06 & 2.60 & 8.1 & 0.87 & & 25.00 & 6.3 & $\mathrm{D}$ & \\
\hline $1143+456$ & $\mathrm{~g}$ & 24.0 & 0.762 & 2.26 & 0.8 & 0.93 & 1.36 & 27.26 & 3.3 & $\mathrm{D}$ & $\ddagger$ \\
\hline $1157+460$ & $\mathrm{~g}$ & 21.3 & 0.742 & 2.88 & $\sim 0.8$ & 0.73 & 1.08 & 27.29 & $\sim 3.2$ & $\mathrm{~T} ?$ & $\ddagger$ \\
\hline $1159+395$ & $\mathrm{~g}$ & 23.4 & 2.37 & 0.83 & 0.05 & 0.22 & 0.75 & 27.57 & 0.2 & $\mathrm{U}$ & \\
\hline $1201+394$ & $\mathrm{G}$ & 19.0 & 0.445 & 1.11 & 2.1 & 0.66 & 1.27 & 26.40 & 7.2 & $\mathrm{D}$ & $\ddagger$ \\
\hline $1204+401$ & $\mathrm{~g}$ & 23.0 & 2.066 & 1.00 & 1.6 & 1.09 & 1.13 & 27.94 & 6.7 & $\mathrm{D}$ & $\ddagger$ \\
\hline $1212+380$ & $\mathrm{~g}$ & 24.0 & $1.5 \mathrm{~K}$ & 1.08 & 0.3 & 1.03 & 1.18 & 27.63 & 1.3 & SR & $\ddagger$ \\
\hline $1216+402$ & $\mathrm{~g}$ & 22.1 & 0.756 & 1.00 & 3.8 & 0.81 & 0.94 & 26.88 & 15.5 & $\mathrm{D}$ & $\ddagger$ \\
\hline $1217+427$ & $\mathrm{E}$ & & & 0.87 & 2.7 & 0.85 & 1.01 & $>26.5$ & {$[11.7]$} & $\mathrm{D}$ & \\
\hline $1220+408$ & $\mathrm{~g}$ & 23.2 & & 1.50 & 4.1 & 0.94 & 1.09 & $>26.7$ & {$[17.6]$} & $\mathrm{Da}$ & $\ddagger$ \\
\hline
\end{tabular}


Table 1. continued

\begin{tabular}{|c|c|c|c|c|c|c|c|c|c|c|c|}
\hline Name & Id & $m_{R}$ & $z$ & $\begin{array}{l}S_{0.4} \\
(\mathrm{Jy})\end{array}$ & $\begin{array}{r}L A S \\
(\operatorname{arcsec})\end{array}$ & $\alpha_{0.4}^{1.4}$ & $\alpha_{4.9}^{8.5}$ & $\begin{array}{r}\log P_{0.4} \\
\left(\mathrm{~W} / \mathrm{Hz} h^{-2}\right)\end{array}$ & $\begin{array}{r}L L S \\
\left(\mathrm{kpc} h^{-1}\right) \\
\end{array}$ & \multicolumn{2}{|c|}{ Morph. } \\
\hline $1225+442$ & $\mathrm{G}$ & 18.2 & $0.22 \mathrm{R}$ & 0.93 & 0.2 & 0.72 & 1.28 & 25.70 & 0.5 & SR & $\bar{\ddagger}$ \\
\hline $1233+418$ & G & 18.4 & $0.25 \mathrm{R}$ & 1.47 & $\sim 2.2$ & 0.59 & 0.72 & 26.00 & $\sim 5.3$ & $\mathrm{~T}$ & $\ddagger$ \\
\hline $1241+411$ & G & 17.7 & 0.259 & 0.80 & $\sim 1.0$ & 0.63 & 0.74 & 25.72 & $\sim 2.6$ & D & $\ddagger$ \\
\hline $1242+410$ & $\mathrm{Q}$ & 19.7 & 0.811 & 2.01 & 0.04 & 0.32 & 0.61 & 27.08 & 0.2 & $\mathrm{U}$ & \\
\hline $1314+453 \mathrm{~A}$ & $\mathrm{~g}$ & 21.8 & 1.544 & 1.75 & 0.16 & 0.64 & 0.83 & 27.77 & 0.7 & $\mathrm{U}$ & \\
\hline $1340+439$ & $\mathrm{E}$ & & & 1.20 & 0.07 & 0.63 & 1.20 & $>26.5$ & {$[0.3]$} & $\mathrm{U}$ & \\
\hline $1343+386$ & $\mathrm{Q}$ & 17.5 & 1.844 & 1.52 & 0.11 & 0.42 & 0.58 & 27.70 & 0.5 & $\mathrm{U}$ & $\ddagger$ \\
\hline $1350+432$ & $\mathrm{~g}$ & 22.1 & 2.149 & 0.83 & 1.6 & 1.28 & 1.38 & 28.00 & 6.5 & $\mathrm{D}$ ? & $\ddagger$ \\
\hline $1432+428 \mathrm{~B}$ & $\mathrm{E}$ & & & 0.93 & 0.04 & 0.01 & 0.78 & $>26.3$ & {$[0.2]$} & $\mathrm{U}$ & $\ddagger$ \\
\hline $1441+409$ & $\mathrm{E}$ & & & 1.68 & 0.1 & 0.43 & 0.89 & $>26.7$ & {$[0.4]$} & $\mathrm{U}$ & \\
\hline $1445+410$ & G & 17.9 & 0.18 & 0.98 & 8.1 & 0.69 & & 25.54 & 15.7 & $\mathrm{D}$ & \\
\hline $1449+421$ & $\mathrm{E}$ & & & 2.37 & 0.08 & 0.81 & 1.44 & $>26.9$ & {$[0.3]$} & $\mathrm{U}$ & \\
\hline $1458+433$ & $\mathrm{~g}$ & 22.3 & 0.927 & 1.08 & 1.6 & 0.72 & 0.93 & 27.08 & 6.9 & $\mathrm{~T}$ & $\ddagger$ \\
\hline $2301+443$ & $\mathrm{~g}$ & & $1.7 \mathrm{~K}$ & 4.04 & 0.5 & 0.95 & 1.49 & 28.34 & 2.1 & $\mathrm{D}$ & \\
\hline $2302+402$ & $\mathrm{E}$ & & & 2.53 & 0.6 & 0.61 & 1.20 & $>26.8$ & {$[2.6]$} & $\mathrm{D}$ & $\ddagger$ \\
\hline $2304+377$ & G & 19.7 & $0.40 \mathrm{R}$ & 2.60 & 0.1 & 0.45 & 1.01 & 26.44 & 0.3 & $\mathrm{U}$ & \\
\hline $2311+469$ & $\mathrm{Q}$ & 17.5 & 0.745 & 4.34 & 2.4 & 0.66 & 0.96 & 27.44 & 9.6 & Dc? & $\ddagger$ \\
\hline $2322+403$ & $\mathrm{E}$ & & & 0.85 & 3.2 & 0.75 & 1.24 & $>26.4$ & [14.0] & $\mathrm{D} ?$ & $\ddagger$ \\
\hline $2330+402$ & $\mathrm{E}$ & & & 1.78 & 0.07 & 0.63 & 0.98 & $>26.7$ & {$[0.3]$} & $\mathrm{U}$ & \\
\hline $2348+450$ & $\mathrm{~g}$ & & 0.978 & 1.80 & 0.2 & 0.70 & 1.15 & 27.36 & 0.9 & SR & \\
\hline $2349+410$ & $\mathrm{Q}$ & 19.2 & 2.046 & 1.38 & 1.2 & 0.90 & 1.22 & 27.99 & 4.7 & $\mathrm{~T}$ & $\ddagger$ \\
\hline $2358+406$ & $\mathrm{E}$ & & & 2.02 & 0.08 & 0.34 & 0.83 & $>26.8$ & {$[0.3]$} & $\mathrm{U}$ & \\
\hline
\end{tabular}

Morphological Classification (last column)

- U (unresolved): $L A S<0.2^{\prime \prime}$;

- SR (Slightly Resolved): $0.2^{\prime \prime}{ }^{\prime} \lesssim A S \lesssim 0.4^{\prime \prime}$;

- D (Double): two distinct components with $\alpha \gtrsim 0.5$;

- Dc: $D$ with a clearly detected core;

- Da: very asymmetric $D$;

- T (Triple): three distinct steep spectrum components;

- scJ (steep core + Jet): elongated structure, sometimes a sequence of knots, very asymmetric in brightness, where the brightest component is at one of the source edges and has a straight steep spectrum;

- fcJ (flat core + Jet): core-jet source, with a flat spectrum core;

- Cx (Complex): complex morphology.

- Column 9: Log of radio luminosity at $408 \mathrm{MHz}$ in $\mathrm{W} / \mathrm{Hz} h^{-2}$; lower limits have been computed for $z=0.5$;

- Column 10: Largest Linear Size, in $\mathrm{kpc} h^{-1}$; values in square brackets ([ ]) are computed assuming $z=1.05$ (see Sect. 3);

- Column 11: morphological classification;

- A $\ddagger$ refers to a note on the source (Sect. 5.6).

The optical identifications on the POSS prints have brought to $12 G(\approx 14 \%)$ and $9 Q(\approx 10 \%)$ brighter than $m_{R} \approx 20.5$. The fraction of such identifications is, within the statistical uncertainties, the one reported by Vigotti et al. (1989) for the whole B3-VLA sample. Deeper optical searches have produced 32 new galaxies and only one additional quasars $(0144+432$, L. Maxfield, private communication). Thirty three objects $(38 \%)$ do not have a redshift yet.

In Fig. 1 we plot the distribution of $\log P_{0.4}$ vs. $z$ for the 3CR sample (Fanti et al. 1995) and for our sources.
To produce the plot, to the sources for which no $z$ is available yet ( + in the figure) a random redshift $>0.5$ has been attributed with the same distribution of the redshifts measured for former $E$ sources. Clearly the new sample is, on average, somewhat deeper than the old one, as might have been expected given the lower flux density limit.

\section{Results}

\subsection{Total intensity parameters}

We considered only source components with peak brightness $S_{\mathrm{p}} \gtrsim 3 \sigma$. Their parameters were determined by means of the task JMFIT, when this was acceptable, i.e. when residuals after model subtraction were comparable to the noise. Slightly extended components were fitted by a two Gaussian model and the solution accepted only if the separation between the two components was larger than about one beam size $(H P B W)$, otherwise one single 
Table 2. Sources excluded from the CSS sample

\begin{tabular}{|c|c|c|c|c|c|c|c|c|c|c|c|}
\hline Name & Id & $m_{R}$ & $z$ & $\begin{array}{l}S_{0.4} \\
(\mathrm{Jy})\end{array}$ & $\begin{array}{r}L A S \\
(\operatorname{arcsec})\end{array}$ & $\alpha_{0.4}^{1.4}$ & $\alpha_{4.9}^{8.5}$ & $\begin{array}{r}\log P_{0.4} \\
\left(\mathrm{~W} / \mathrm{Hz} h^{-2}\right)\end{array}$ & $\begin{array}{r}L L S \\
\left(\operatorname{kpc} h^{-1}\right)\end{array}$ & \multicolumn{2}{|c|}{ Morph. } \\
\hline $0032+423$ & Q & 17.5 & 1.588 & 0.92 & $\sim 6.0$ & 0.78 & 1.23 & 27.55 & $\sim 26.0$ & scJ & \\
\hline $0130+381$ & $\mathrm{G}$ & 19.0 & $0.4 \mathrm{R}$ & 1.07 & $\sim 7.0$ & 0.73 & 0.56 & 26.28 & $\sim 22.5$ & Dc & $\ddagger$ \\
\hline $0244+377$ & $\mathrm{E}$ & & & 1.73 & $\sim 11$ & 0.69 & 1.15 & $>26.7$ & {$[\sim 47.0]$} & $\mathrm{Da}$ & $\ddagger$ \\
\hline $0827+378$ & Q & 17.6 & 0.914 & 5.17 & $\sim 9.0$ & 0.65 & 0.62 & 27.74 & $\sim 38.0$ & fcJ? & $\ddagger$ \\
\hline $0900+428$ & G & 20.7 & $0.33 \mathrm{R}$ & 2.65 & $\sim 15.0$ & 0.60 & 0.27 & 26.50 & $\sim 45.0$ & Dc & $\ddagger$ \\
\hline $0922+422$ & Q & 17.9 & 1.75 & 0.97 & 5.5 & 1.37 & 1.28 & 27.78 & 23.0 & scJ & $\ddagger$ \\
\hline $1253+432$ & $\mathrm{~g}$ & & 2.32 & 1.42 & 5.4 & 0.95 & 0.87 & 28.11 & 21.5 & $\mathrm{Da}$ & \\
\hline $2305+404$ & $\mathrm{~g}$ & & 2.14 & 0.99 & 20.0 & 1.10 & 1.51 & 27.95 & 82.0 & Dc & $\ddagger$ \\
\hline $2321+423$ & $\mathrm{~g}$ & 23.2 & 0.918 & 2.07 & $\sim 11$ & 0.66 & 1.13 & 27.30 & $\sim 44.0$ & $\mathrm{D}$ & $\ddagger$ \\
\hline $0739+397 \mathrm{~B}$ & $\mathrm{Q}$ & 18.2 & 1.70 & $\approx 0.6$ & 2.0 & $\approx 0.2$ & -0.36 & $\overline{27.50}$ & 8.6 & fcJ & $\ddagger$ \\
\hline $0945+408$ & Q & 17.6 & 1.252 & 2.45 & 4.0 & 0.33 & -0.06 & 27.60 & 17.0 & fcJ & \\
\hline
\end{tabular}

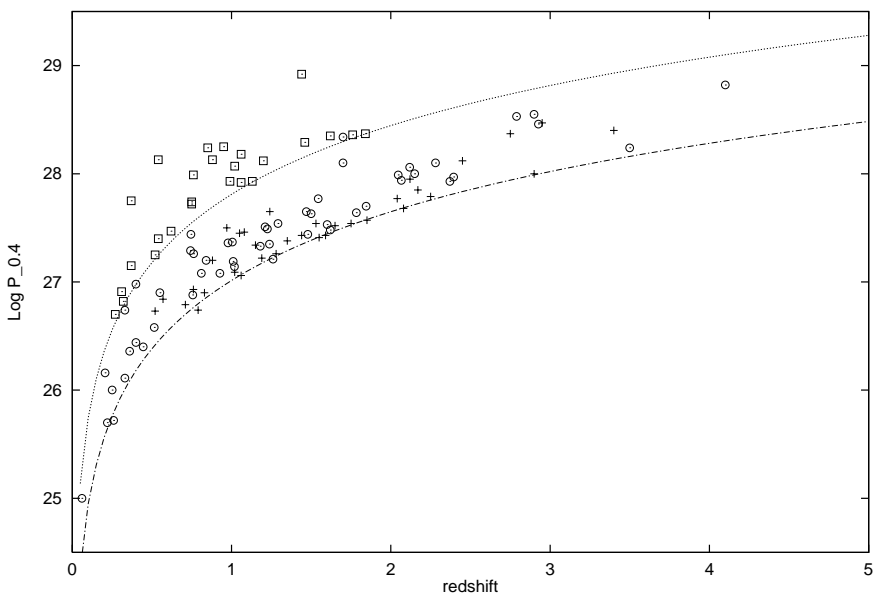

Fig. 1. $P-z$ plane for the $3 \mathrm{CR}$ (boxes) and the B3-VLA samples $[($ circles $)$ and (plus) are data with and without $z$; see text]. The lines represent the $P-z$ relations for the $408 \mathrm{MHz}$ flux density limits of $0.8 \mathrm{Jy}$ (B3-VLA) and $5 \mathrm{Jy}$ (approximately 3CR)

extended feature was considered. When a sub-structure was too extended to be reliably fitted by a multiple Gaussian model, its flux density was determined by integration of its brightness with TVSTAT, and its centroid position and diameter were estimated from the image contour plot. Note that, in this case, the angular size is roughly twice the conventional $H P W$. In this last case data in Tables 1 to 3bis (Sect. 5.4) are marked by a " ".

In order to check whether our flux densities, measured either from Gaussian fits or from integration, may be underestimated due to resolution effects, we examined the visibility amplitudes of the shortest baselines of each source. Particular care has been paid at $8.5 \mathrm{GHz}$ (shortest baseline $\lesssim 12 \mathrm{k} \lambda$ ) where the risk of missing flux is the largest. After this exam we are confident that any missing flux density is, on average, definitely less than $3 \%$ even in the well resolved source components.

We have then compared our total flux densities at 1.5 and $4.9 \mathrm{GHz}$ with lower resolution measurements from the literature (Figs. 2a,b). Precisely: for the $1.5 \mathrm{GHz}$ data we used the NVSS survey $(1.4 \mathrm{GHz})$ after having derived the flux densities from the original images by means of the task TVSTAT; at $4.9 \mathrm{GHz}$ the comparison data are from the database $(D B)$ by Vigotti et al. (1999). Using the 29 $U$ and $S R$ sources we find:

$$
\begin{aligned}
& S_{\mathrm{B} 3-\mathrm{VLA}}^{1.5} / S_{\mathrm{NVSS}}^{1.4}=0.94 \pm 0.01 \quad \sigma=0.07 \\
& S_{\mathrm{VLA}}^{4.9} / S_{\mathrm{DB}}^{4.9} \quad=1.03 \pm 0.01 \quad \sigma=0.05 \text {. }
\end{aligned}
$$

The apparent excess of $S_{\mathrm{NVSS}}$ over $S_{\mathrm{B} 3-\mathrm{VLA}}$ is largely accounted for by the slight difference in the observing frequencies (1.4 and 1.465 respectively). For instance, an average spectral index $\alpha \approx 1$, as found for this sample (Sect. 6.2), would give $S_{\mathrm{B} 3-\mathrm{VLA}} / S_{\mathrm{NVSS}}=0.95$. The flux densities at $4.9 \mathrm{GHz}$ from the database, instead, seem to be some $3 \%$ lower than our $V L A-A$ measurements. If we do the same comparison using only the extended sources, we find average ratios which are less than $\approx 0.02$ smaller than the previous ones. This supports our previous statement that the extended sources suffer only very minor flux density losses.

At $8.5 \mathrm{GHz}$, since no systematic flux density measurements are available in the literature, we attempted a comparison of our VLA data with those obtained by linear interpolation of the flux densities at $4.9 \mathrm{GHz}$ and at $10.6 \mathrm{GHz}$ by Vigotti et al. (1999). We get $S_{\text {VLA }}^{8.5} / S_{\text {interp. }}^{8.5} \approx$ $1.12 \pm 0.01$. Such a high flux density ratio is due to the fact, well visible in the compilation of the source total spectra from the database by Vigotti et al. (1999), that in this frequency range the spectra are clearly down curved at high frequencies. Hence the linear interpolation is not adequate.

\subsection{Polarization parameters}

Besides the total intensity $(I)$, we produced also images for the two Stokes parameters $Q$ and $U$ at both 8.5 and $4.9 \mathrm{GHz}$. From them the polarized intensity $(P)$ images were obtained as $P_{i j}=\sqrt{Q_{i j}^{2}+U_{i j}^{2}}$, ij being the image pixel coordinates. The effect of the noise on $P$ images is always positive and produces an upward bias (e.g. Simmons $\&$ Stewart 1985). The data follow a Rice distribution which approaches a Gaussian statistics at high signal-tonoise ratios (see Vinokur 1965). For lower signal-to-noise 

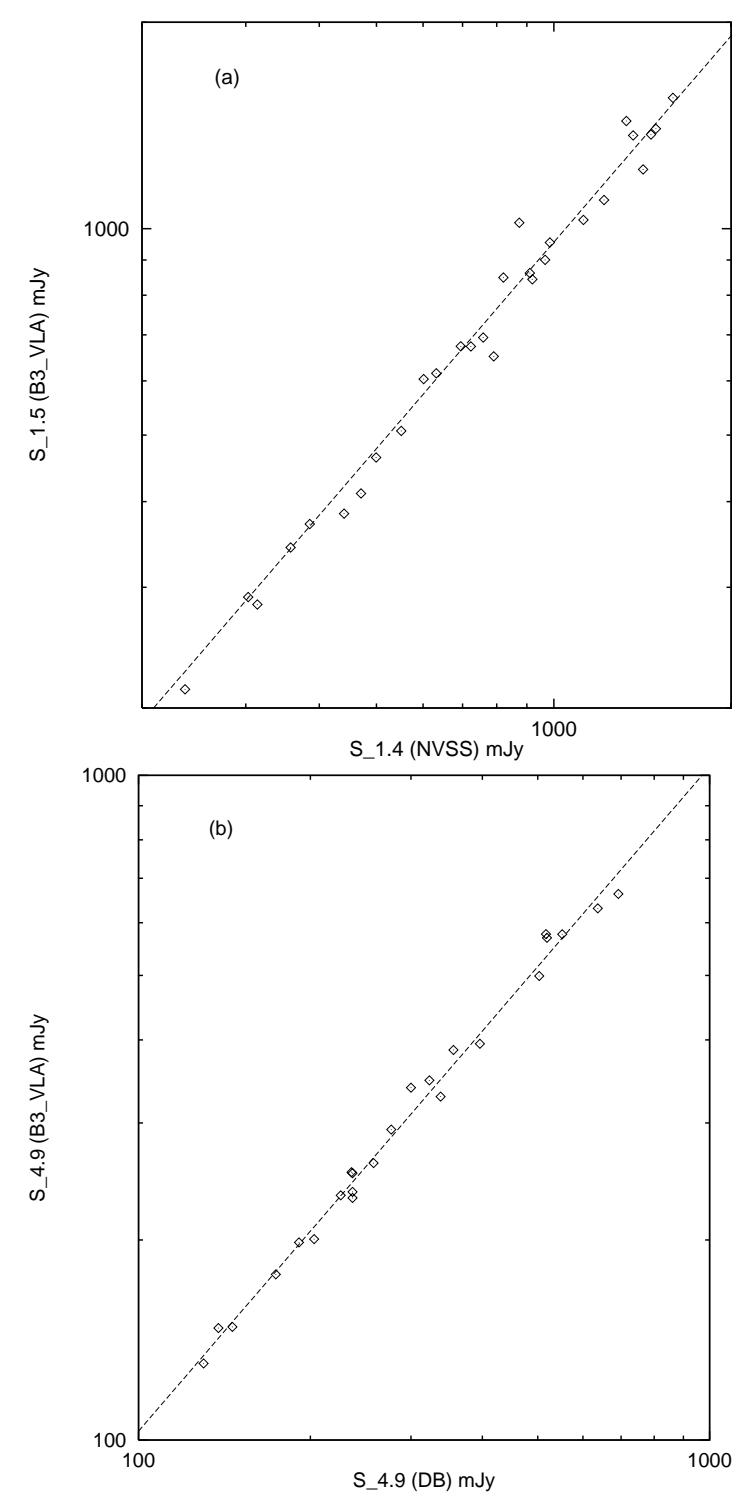

Fig. 2. Flux density comparison, for $U$ and $S R$ sources, at: a) $1.465 \mathrm{GHz}$, and b) $4.9 \mathrm{GHz}$. The straight lines have slopes 0.94 and 1.03 and their meaning is discussed in the text

ratios a correction has to be applied. This is estimated to be around $10 \%$ for observed $P_{i j} \sim 2.5 \sigma$ and asymptotes to zero for increasing $P_{i j} / \sigma$. We used the AIPS option which corrects for this bias. In addition, we blanked out all the points for which observed $P_{i j}<3 \sigma$, to avoid possible misuse of low signal-to-noise polarization data. Therefore the correction applied to the remaining data points is generally small. From here on, when talking of polarization data, we always refer to the images de-biased and blanked of noise dominated data points.

Finally we produced fractional polarization image as: $p_{i j}=P_{i j} / I_{i j}$

excluding pixels for which $I_{i j}<3 \sigma$ to avoid spuriously high values of $p_{i j}$, often found at the edge of the components.

For each source component the average fractional polarization and the associated mean position angle of the electric $(E)$ vector ( $m$ and $\chi$ in Table 3 ) were obtained. In order to do this we set, around each component on $I$ images, a rectangular area or "box" which covered the same sky region at the two frequencies and for each Stokes parameter $(Q, U$, etc.). We then computed the component $Q$ and $U$ flux densities as:

$S_{\mathrm{Q}}=\sum_{i j} Q_{i j} / \Omega_{\mathrm{b}} \quad$ and $\quad S_{\mathrm{U}}=\sum_{i j} U_{i j} / \Omega_{\mathrm{b}}$

(where $\Omega_{\mathrm{b}}$ is the beam area) and the position angle of the electric vector as:

$\chi=\frac{1}{2} \tan ^{-1}\left(\frac{S_{\mathrm{U}}}{S_{\mathrm{Q}}}\right)$.

To compute $m$ two approaches are generally found in the literature (e.g. Garrington et al. 1991):

1) vector mean fractional polarization $m_{\mathrm{v}}=\frac{\sqrt{S_{U}^{2}+S_{Q}^{2}}}{S_{T}}$ where $S_{\mathrm{T}}$ is the total component flux density derived from the $I$ image by means of JMFIT or TVSTAT (Table 3 ). This method has the disadvantage that any rotation of the polarization vector within the box reduces the fractional polarization. In spite of the fact that we tried to decompose a source into components as much as reasonable, there are components, unresolved or barely resolved in total intensity, in which clearly $Q$ or $U$ or both change sign within the chosen box (see notes to the sources in Sect. 5.6).

2) scalar mean fractional polarization $m_{\mathrm{s}}=\frac{S_{\mathrm{P}}}{S_{\mathrm{T}}}$ where $S_{\mathrm{P}}=\sum_{i j} P_{i j} / \Omega_{b}$. This choice has the advantage of being insensitive to electric vector rotations and hence produces a better signal-to-noise ratio. We cannot use this approach, however, since the blanking we applied to the $P$ images introduces an underestimate (up to $\approx 30 \%$ for polarized flux densities of 1-2 mJy) of the polarization flux densities and therefore of $m_{\mathrm{s}}$.

Another possible approach is the:

3) average fractional polarization $m=\frac{\sum_{i j} p_{i j}}{M}$

where $M$ is the number of points remained in the box after blanking. After a number of tests, we considered that the last one is the best approach as the values of $p_{i j}$ are quite stable across each component and $m$ is therefore a robust estimate of the percentage polarization. This is a scalar estimate but it is not affected by the lack of points due to blanking of $P_{i j}$. In Table 3 we give this value (in percentage). Our tests also show that, on average, in our sample $m \approx(2.0 \pm 0.4) m_{\mathrm{v}}$.

\subsection{Error estimates}

The rms noise error $\left(\sigma_{I}\right.$, in mJy/beam) has been determined for each field using TVSTAT in "empty" regions on $I$ images. Then the noise error on the total flux density is obtained as:

$\sigma_{\mathrm{T}}=\sigma_{I} \sqrt{N}$ 
where $N$ is the number of independent beam areas through the component. We preferred to adopt this rather than the formal error provided by JMFIT, which merely represents the goodness of the mathematical fit. To derive the total flux density errors one has to add in quadrature the calibration uncertainty which, as said in Sects. 3 and 4 , is $\approx 5 \%$ at $1.5 \mathrm{GHz}$ and $\approx 3 \%$ at high frequencies.

The noise on the $Q$ and $U$ images turned out to be the same $\left(\sigma_{U Q}\right)$ and in the majority of cases not much different from $\sigma_{I}$. Then the errors on $\chi$ can be estimated as follows:

$\sigma_{\chi}=\frac{1}{2} \frac{\sigma_{U Q} \sqrt{N^{\prime}}}{\sqrt{S_{Q}^{2}+S_{U}^{2}}}=\frac{1}{2} \frac{\sigma_{U Q} \sqrt{N^{\prime}}}{m_{\mathrm{v}} S_{\mathrm{T}}} \approx \frac{\sigma_{\mathrm{UQ}} \sqrt{N^{\prime}}}{m S_{\mathrm{T}}} \times 1.3$.

Here we have put $2 m_{\mathrm{v}}=m$ (Sect. 5.2) and have accounted for the uncertainty on this statistical relation. $N^{\prime}$ is the number of independent beam areas in the polarized region, which can be $<N$ (see Fig. 3).

The error on $m$ is given by:

$\sigma_{m}=\frac{\sigma_{P}}{S_{\mathrm{T}}} \sqrt{1+m^{2}\left(\frac{\sigma_{I}}{\sigma_{P}}\right)^{2}} \sqrt{N^{\prime}} \approx \frac{\sigma_{I}}{S_{\mathrm{T}}} \sqrt{1+m^{2}} \sqrt{N^{\prime}}$

where $\sigma_{P}=\sigma_{U Q}$ has been assumed $\approx \sigma_{I}$. For $m \lesssim 0.4$ (or $40 \%$ ) this expression can be approximated with the standard formula (see e.g. Garrington et al. 1991)

$\sigma_{m}=\frac{\sigma_{I}}{S_{\mathrm{T}}} \sqrt{N^{\prime}}$

with an accuracy better than $8 \%$. Formal $1 \sigma$ errors for $\chi$ and $m$, for a $10 \mathrm{mJy}$ point source with $10 \%$ polarization, are $\sim 4^{\circ}$ and $<1 \%$, and scale as $1 / S_{\mathrm{T}}$. The actual uncertainties, however, are likely to be larger mainly due to the indetermination in defining the box within which the measures are obtained.

\subsection{Parameters of the radio components}

Parameters of the source components are presented in Table 3. Of this table we show only a few lines as an example. The table appears in its entirety only at the CDS. Parameters for the sources not belonging to the complete sample are also given in Table 3 bis (only available at the CDS $)^{3}$. The content of both tables is the following:

- Column 1: source name; a " $\ddagger$ " refers to a source note (Sect. 5.6);

- Columns 2 \& 3: component right ascension and declination (equinox B1950.0) as measured at $8.5 \mathrm{GHz}$, in RA order. A " " refers to diffuse or too extended components for which the results of JMFIT were not satisfactory. They represent the approximate component centroid and have been estimated on the image contour plots (Sect. 5.1), usually at $4.9 \mathrm{GHz}$ where extended features are better visible;

\footnotetext{
${ }^{3}$ Both postscript and ASCII versions of either table can be retrieved at the CDS via anonymous ftp to cdsarc.u-strasbg.fr $(130.79 .128 .5)$ or via

ftp://cdsweb.u-strasbg.fr/cgi-bin/qcat?J/A+A/369/380
}

- Columns 4, 5, 6: flux densities at 8.5, 4.9 and $1.5 \mathrm{GHz}$ (1st line); percentage polarization $m$ (2nd line) and polarization angle $\chi$ (3rd line) at 8.5 and $4.9 \mathrm{GHz}$. A " $\star$ " by any of these values means that it is the total (intensity or polarization) of the group of components enclosed between $\uparrow$ and $\downarrow$; (e.g. 0128+394 in Table 3). A " $\diamond "$ by the polarization data, e.g. first two components of $0128+394$, means that it was not possible to separate the components in the polarization image, therefore the reported values of $m$ and $\chi$ refer to the two together. A "_" means no available data. Total intensity data are from JMFIT except when marked by a " " (e.g. bridge of $0144+432$ in Table 3 ). Upper limits on $m$ are the largest value between the percentage polarization obtained from the ratio $3 \sigma / S_{\mathrm{T}}$ and three times the instrumental polarization (Sect. 4). Hence upper limits on $m$ are always $\geq 1 \%$;

- Column 7: high frequency spectral index $\left(S \propto \nu^{-\alpha}\right)$ computed between 8.5 and $4.9 \mathrm{GHz} ; \alpha$ followed by an " $a$ " (for average) is the 3 -point spectral index computed as linear interpolation of the $\log S-\log \nu$ relation, from 1.5 to $8.5 \mathrm{GHz}$. To compute these spectral indices, multiple components have been summed together when they are not resolved at the lower frequencies. In a few cases " $c x_{-} a$ " means a complex three-point spectrum. A "_-" means no available or reliable data;

- Columns 8, 9, 10: deconvolved major and minor component angular size (arcsec) and major axis position angle (p.a., degree) at $8.5 \mathrm{GHz}$, generally obtained by JMFIT. When marked by a " " (e.g. bridge of $0144+432$ in Table 3) they have been estimated on the image contour plots, usually at $4.9 \mathrm{GHz}$. All component diameters, formal result of JMFIT, are reported in these columns, even if they are somewhat smaller than the beam, and then are little significant. These components are to be intended as "unresolved" by our VLA observations.

\subsection{Source images}

Images at the two frequencies of 4.9 and $8.5 \mathrm{GHz}$ are presented in Fig. 3 for the sources with angular size $\gtrsim 0.25^{\prime \prime}$, in RA order and with the pair of frequencies next to each other for every source. The angular scale is the same for all the sources: this provides immediate information on the relative angular extension allowing to judge directly which are the large and which are the small objects in the sample. We do not show the $1.5 \mathrm{GHz} V L A-A$ images, as not much structure is seen in them, except for the three sources not observed at 4.9 and $8.5 \mathrm{GHz}$ (Fig. 4). The sources we have excluded from the initial selection (Table 2) are reported in Fig. 5. For two of these we also show the $1.5 \mathrm{GHz}$ images.

For each image we give the following information on the plot itself:

a) Peak flux density in mJy/beam;

b) First contour intensity (f.c.) in $\mathrm{mJy} / \mathrm{beam}$; corresponding to $\approx 3 \sigma$ of the noise; contours increase by a factor 2; 
Table 3. Observational parameters for the B3-VLA CSS sources

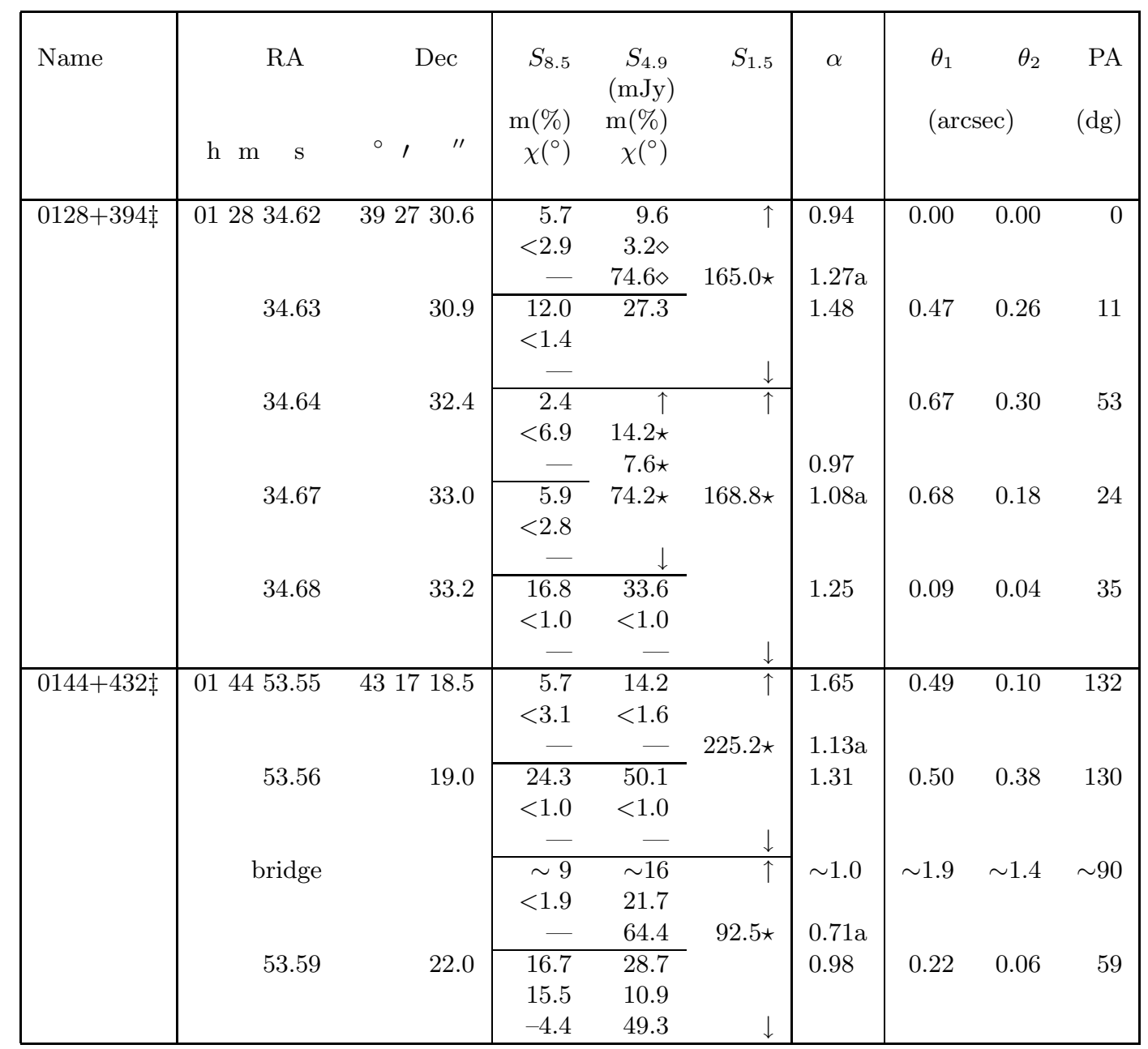

c) Beam size, represented by the ellipse in the lower left corner;

d) Percentage polarization scale by means of a segment, on the top left corner, whose length corresponds to the indicated percentage polarization. In most cases it corresponds to a $10 \%$ polarization, and it allows to judge immediately the low and high polarization components. In any case the percentage polarization scale for each source is the same at both frequencies to allow a visual estimate of the amount of depolarization;

e) The vectors superimposed to the $I$ contours show the percentage polarization (on the indicated scale) and the position angle of the $\boldsymbol{E}$ vector. Note that, due to blanking (Sect. 5.2) no vectors are shown when $P_{i j}<3 \sigma$, otherwise vectors are always plotted even (in few cases) if the percentage polarization of the whole component is not significant and we have given upper limits in Table 3.

\subsection{Notes on individual sources}

\section{Complete sample}

- 0034+444: A large variation of the position angle of the

$\boldsymbol{E}$ vector is seen within the northern component.
- 0039+398: The weak component at $\mathrm{RA}=33.93^{\mathrm{s}}$ and $\mathrm{DEC}=23.1^{\prime \prime}$, based on its location, is a candidate for the source core. However its spectral index is too uncertain to confirm this. The bridge between the two main components, well visible in the $4.9 \mathrm{GHz}$ image, is also present in a lower resolution image (not shown) at $8.5 \mathrm{GHz}$.

$-0041+425$ : A large variation of the position angle of the $\boldsymbol{E}$ vector is seen within the southern component.

$-0049+379$ : There is a large asymmetry in fractional polarization between components at $8.5 \mathrm{GHz}$. The northern one is strongly depolarized.

- 0120+405: A large difference is seen in the Faraday Rotation of the two components.

- 0123+402: A large Faraday Rotation is present in the southernmost component.

$-0128+394:$ The component at $\mathrm{RA}=34.64^{\mathrm{s}}$ and $\mathrm{DEC}=$ $32.4^{\prime \prime}$, based on its location, might be the source core. Since it is not resolved from the lobe at $4.9 \mathrm{GHz}$, we can only set an upper limit of $\approx 2 \mathrm{mJy} /$ beam at the position of the $8.5 \mathrm{GHz}$ coordinates. 

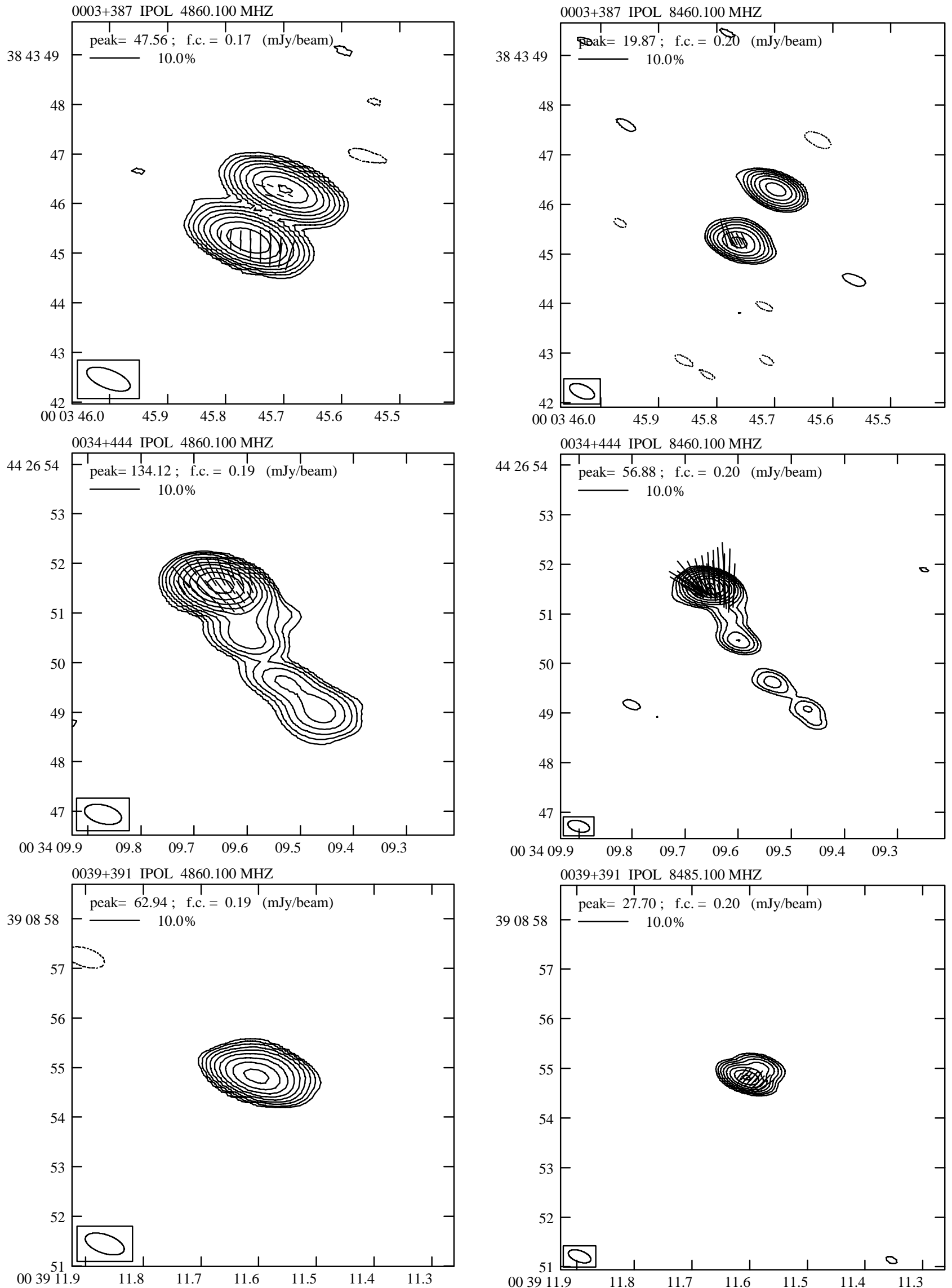

Fig. 3. Images of B3-VLA CSS sources at 4.9 (left) and 8.5 (right) $\mathrm{GHz}$ 

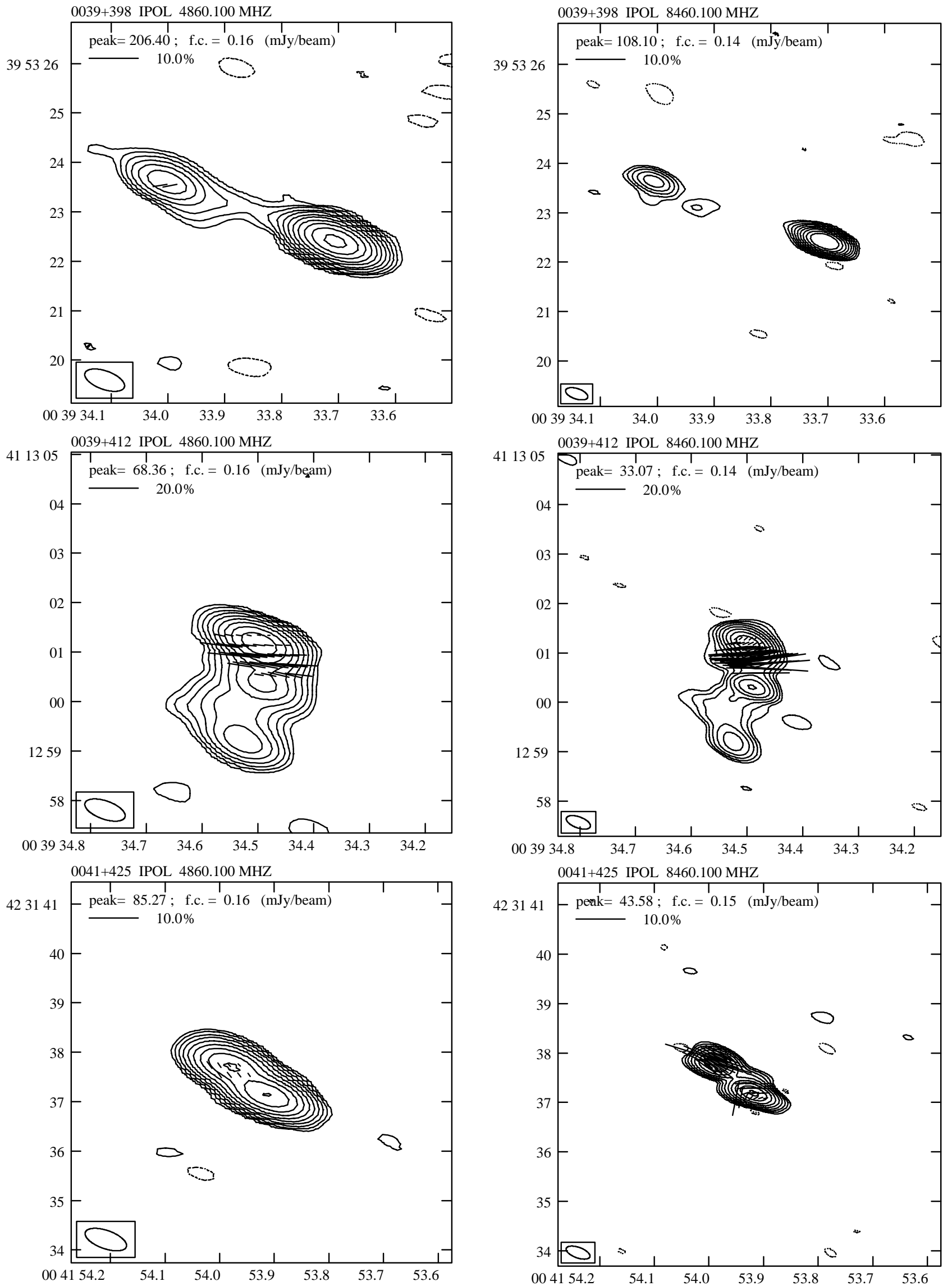

Fig. 3. continued 

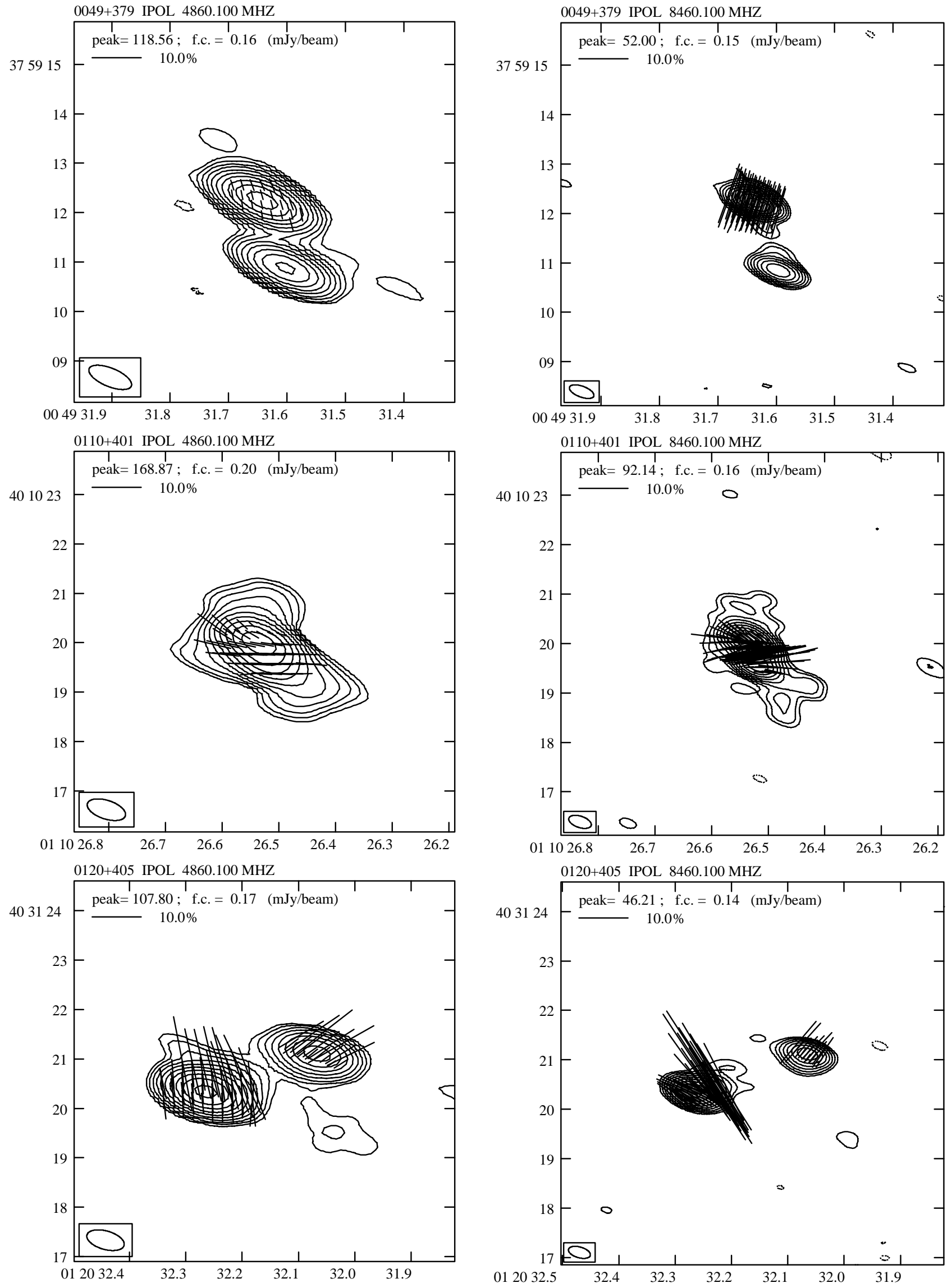

Fig. 3. continued 

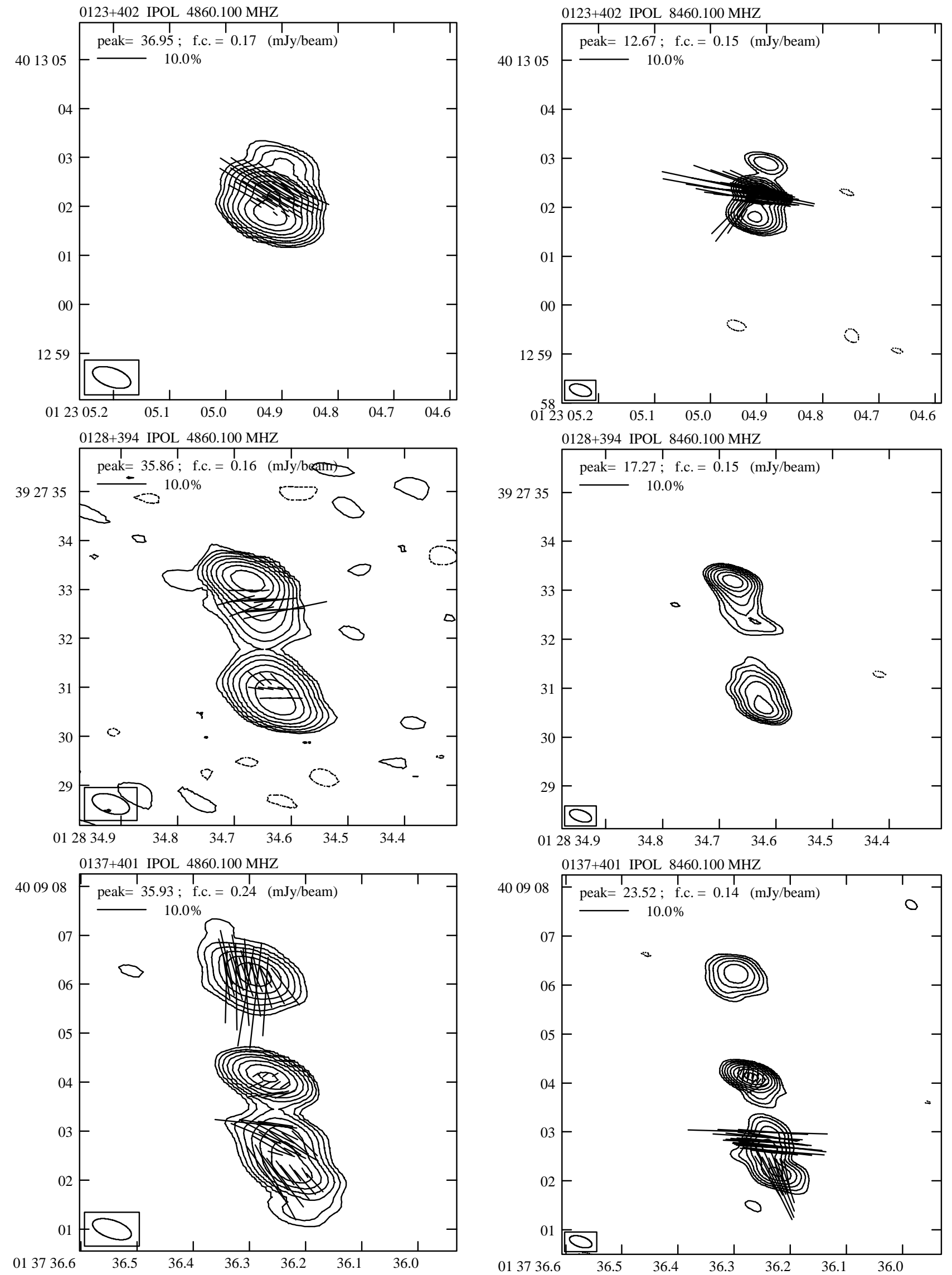

Fig. 3. continued 

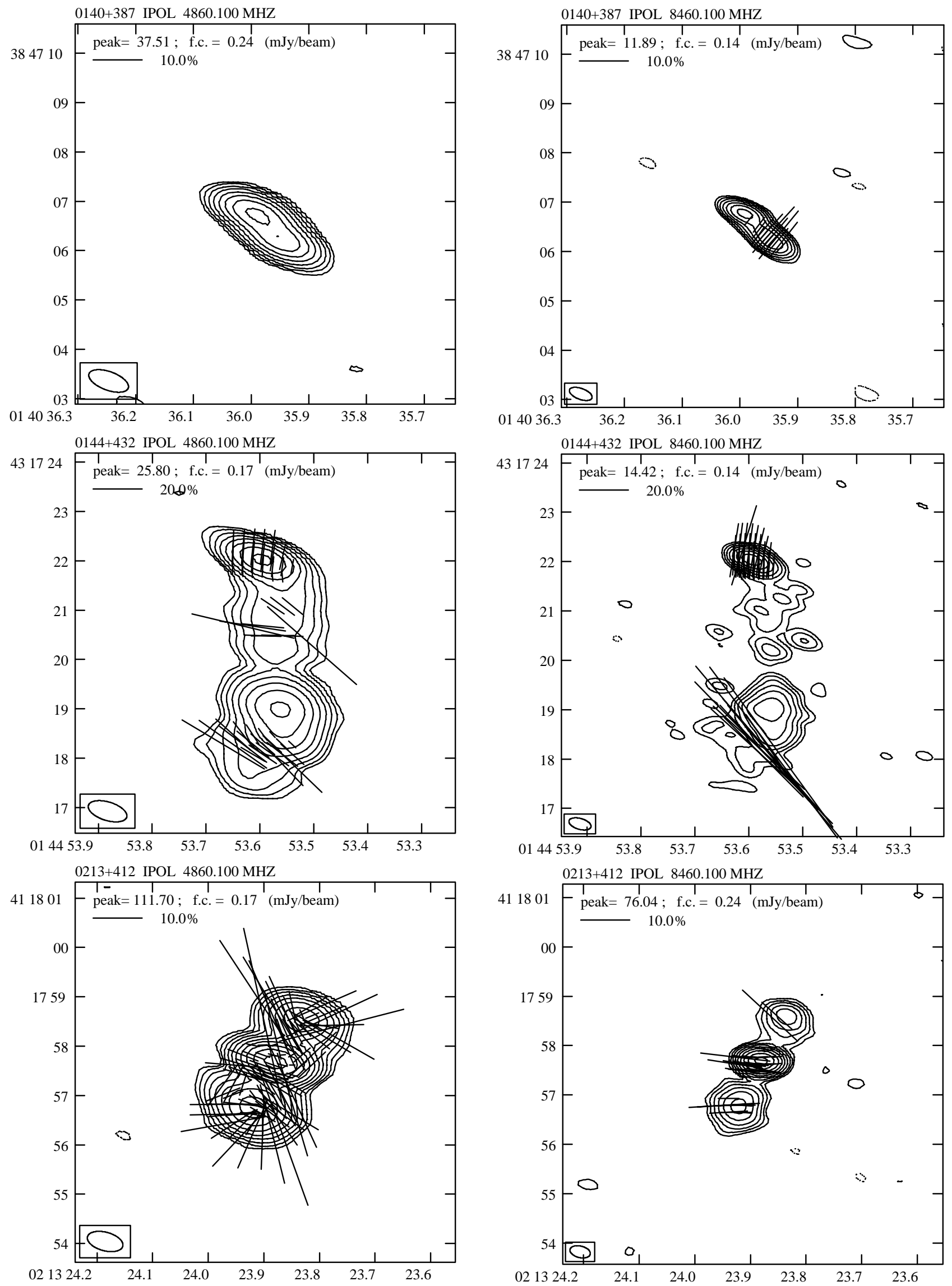

Fig. 3. continued 

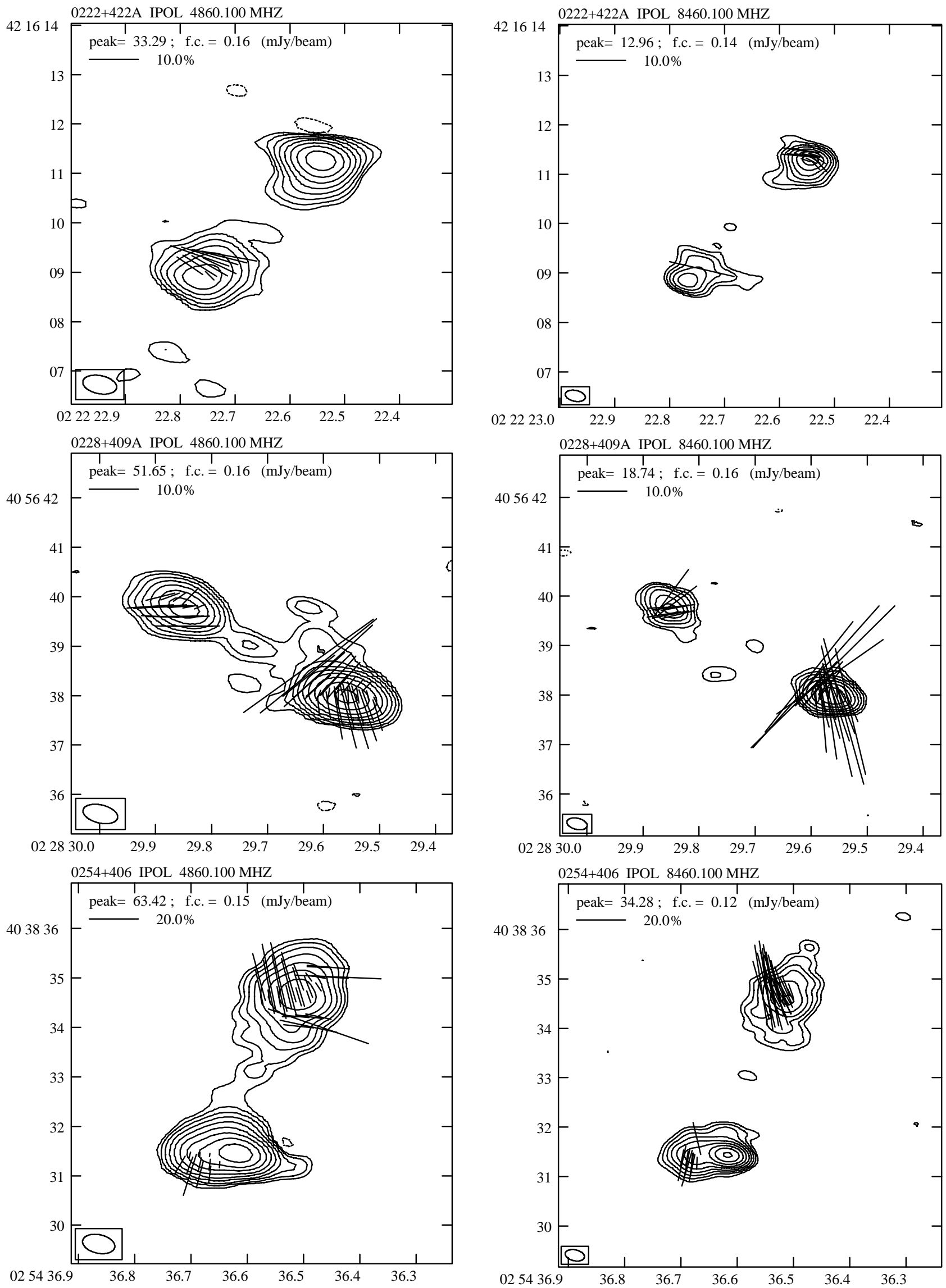

Fig. 3. continued 

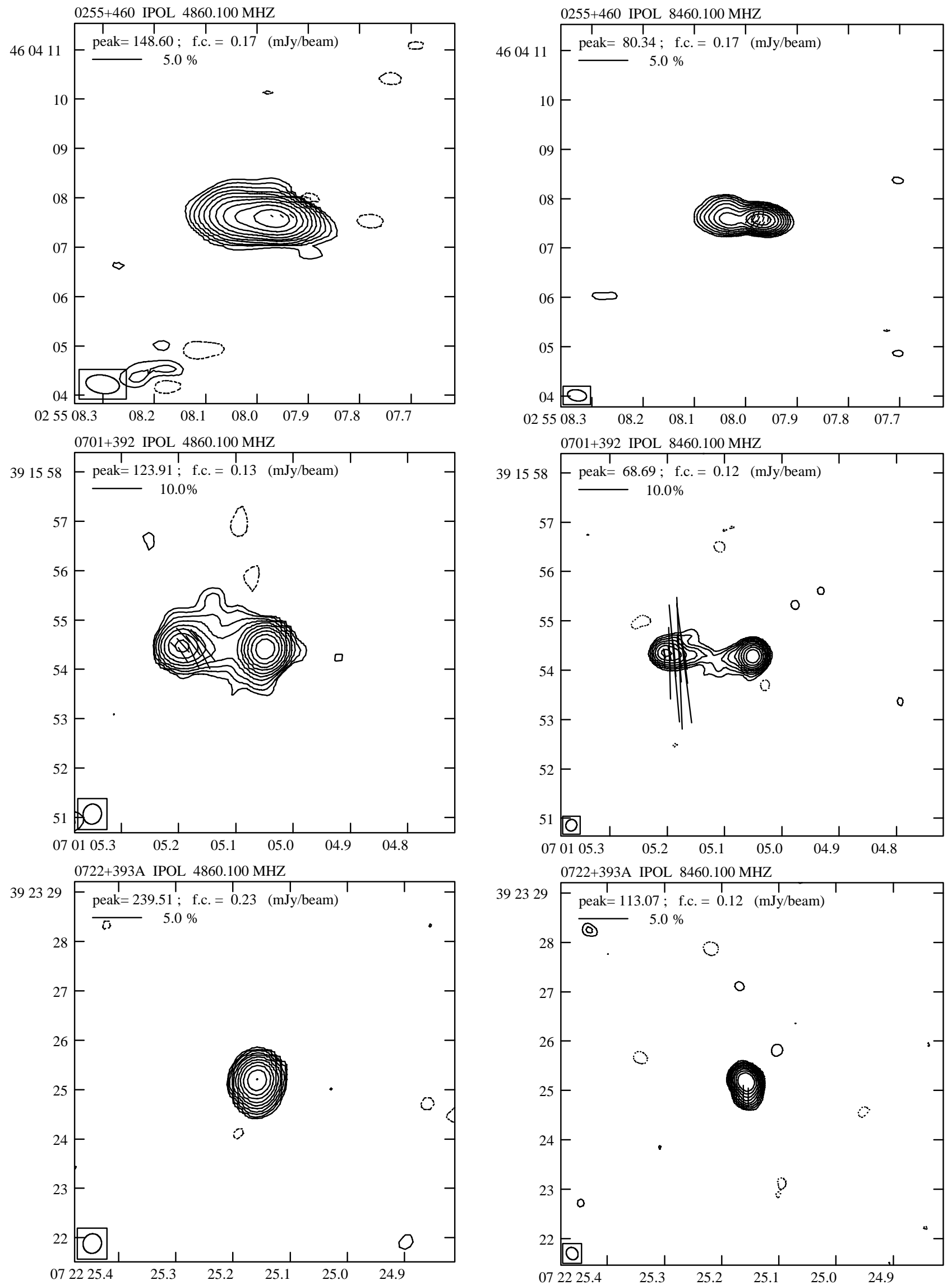

Fig. 3. continued 

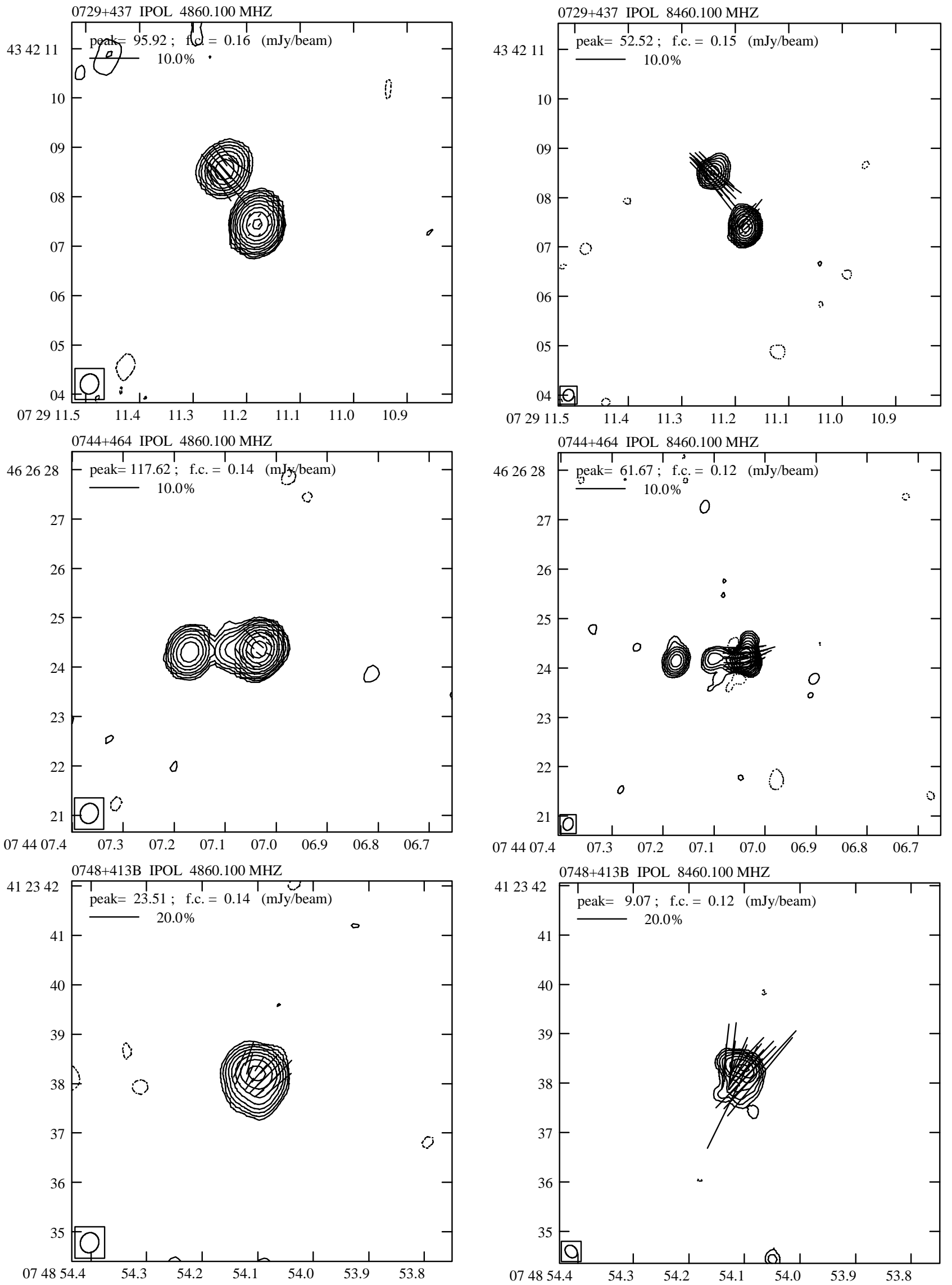

Fig. 3. continued 

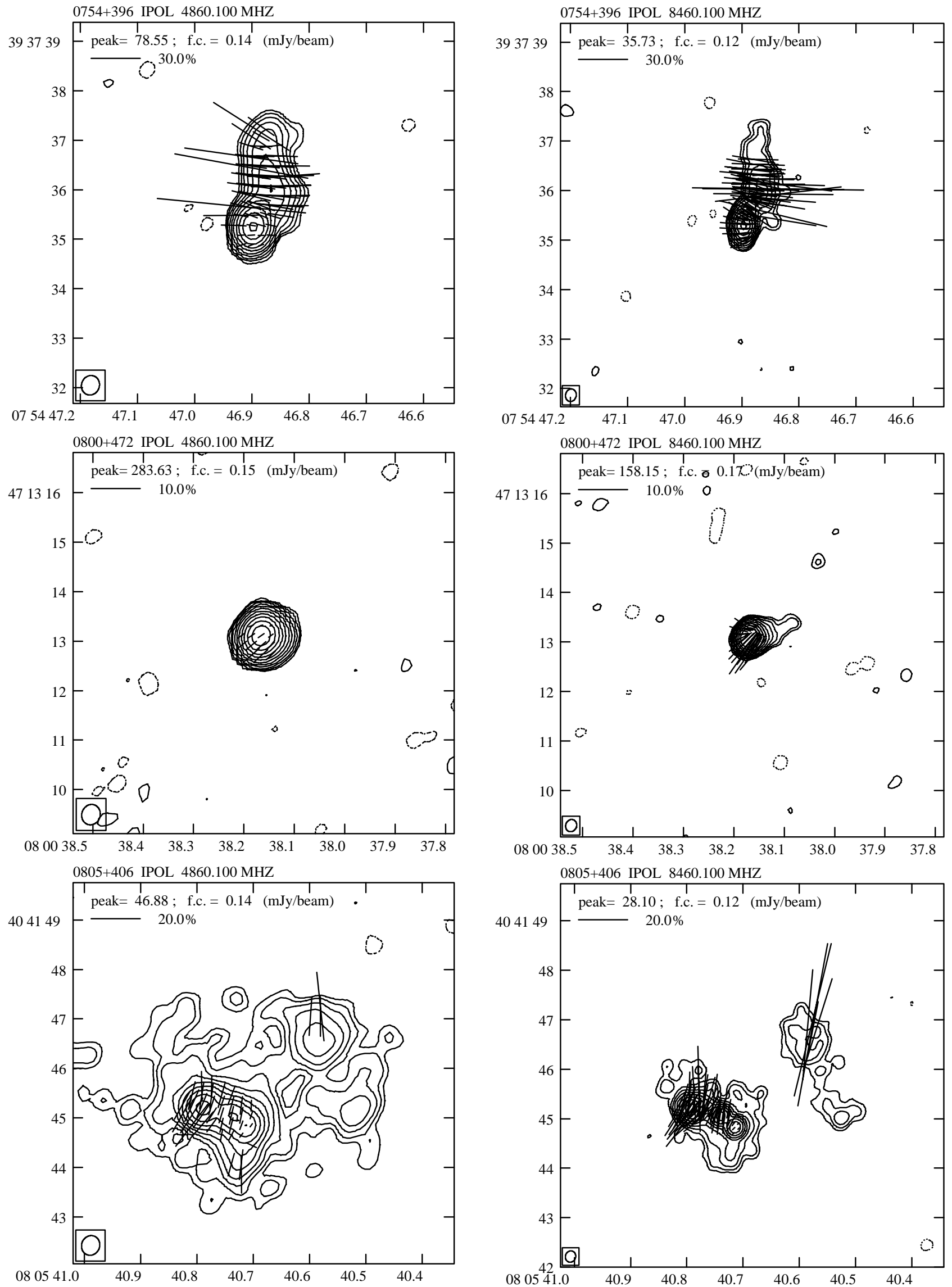

Fig. 3. continued 

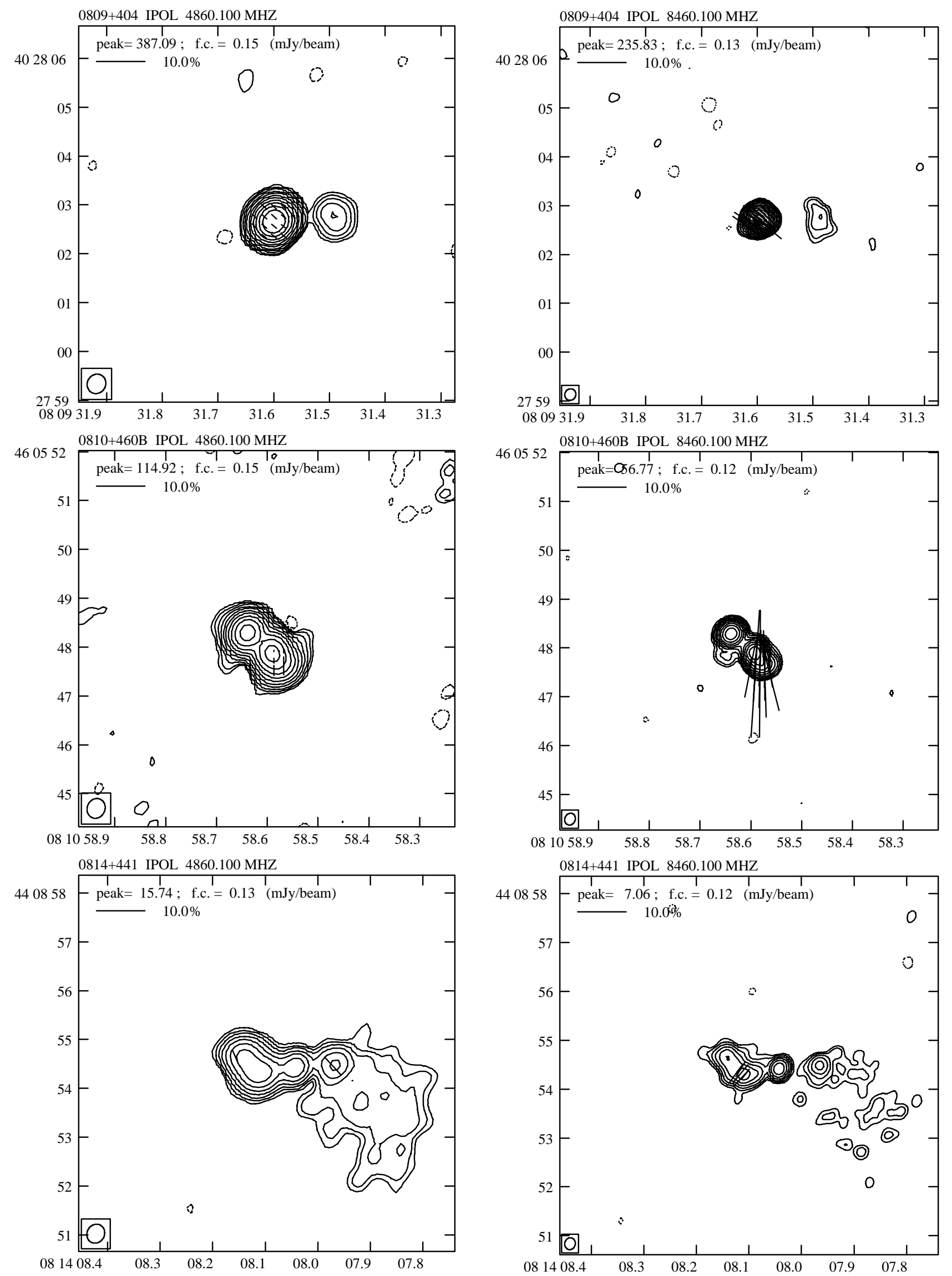

Fig. 3. continued 

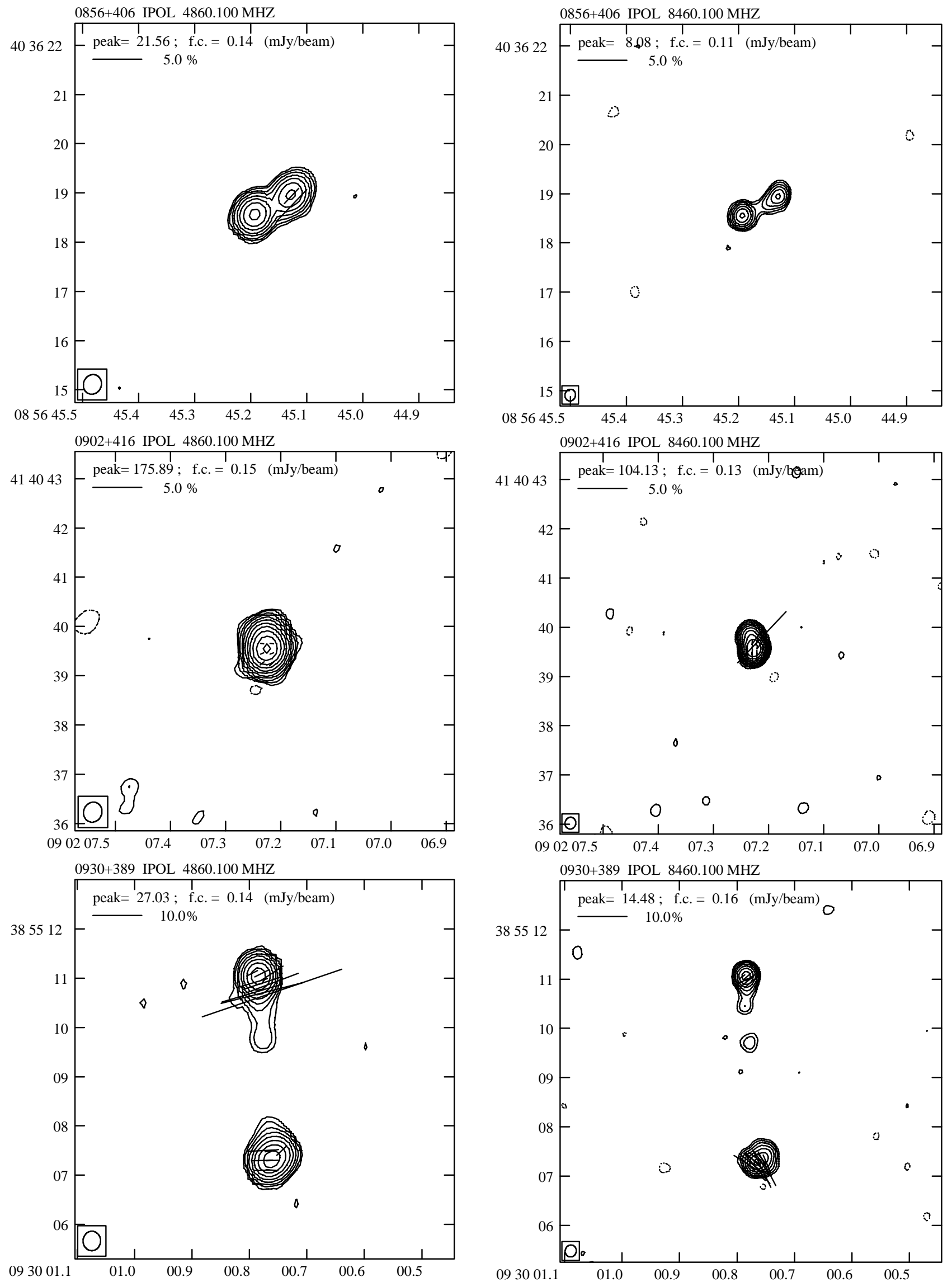

Fig. 3. continued 

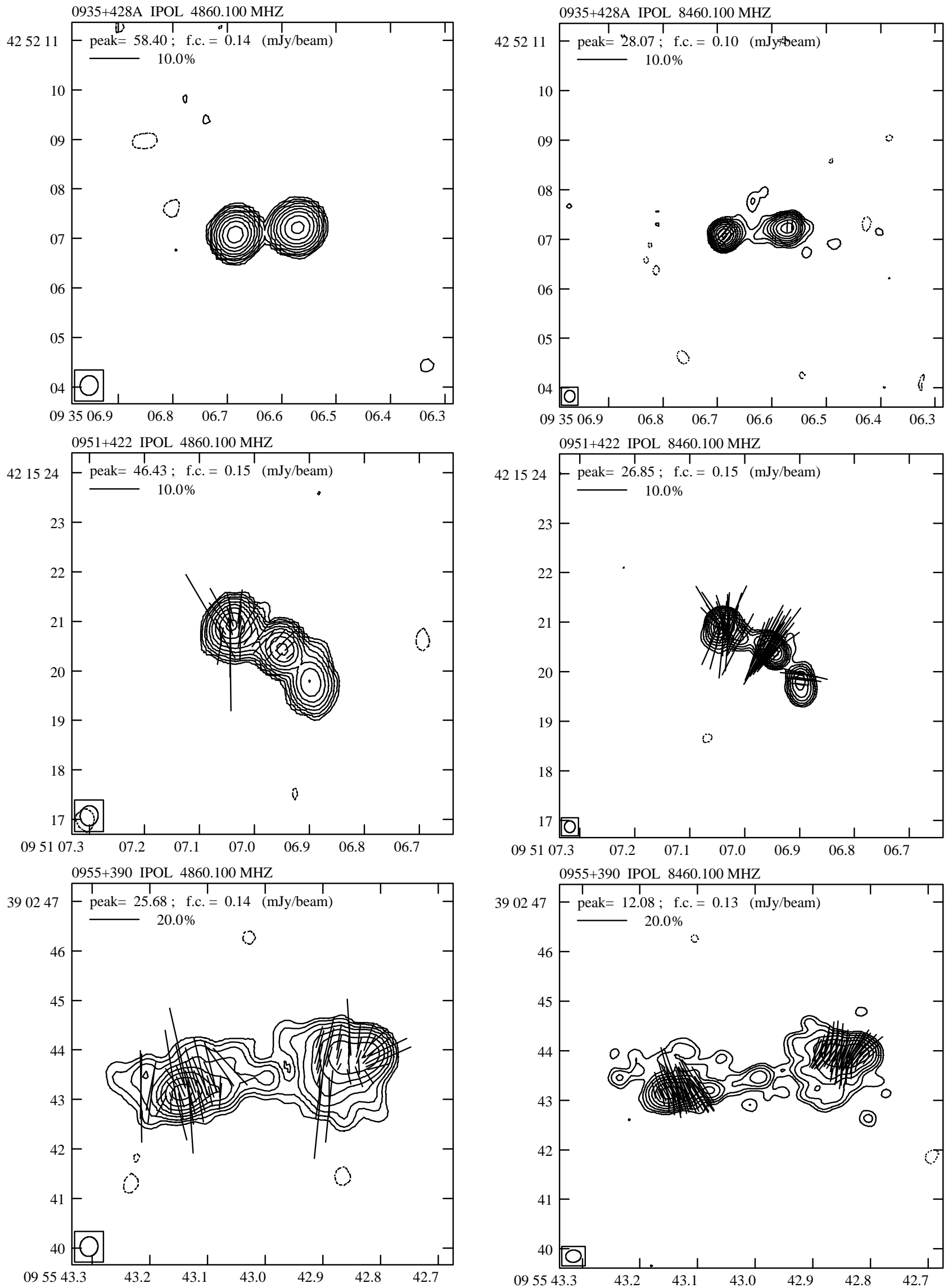

Fig. 3. continued 

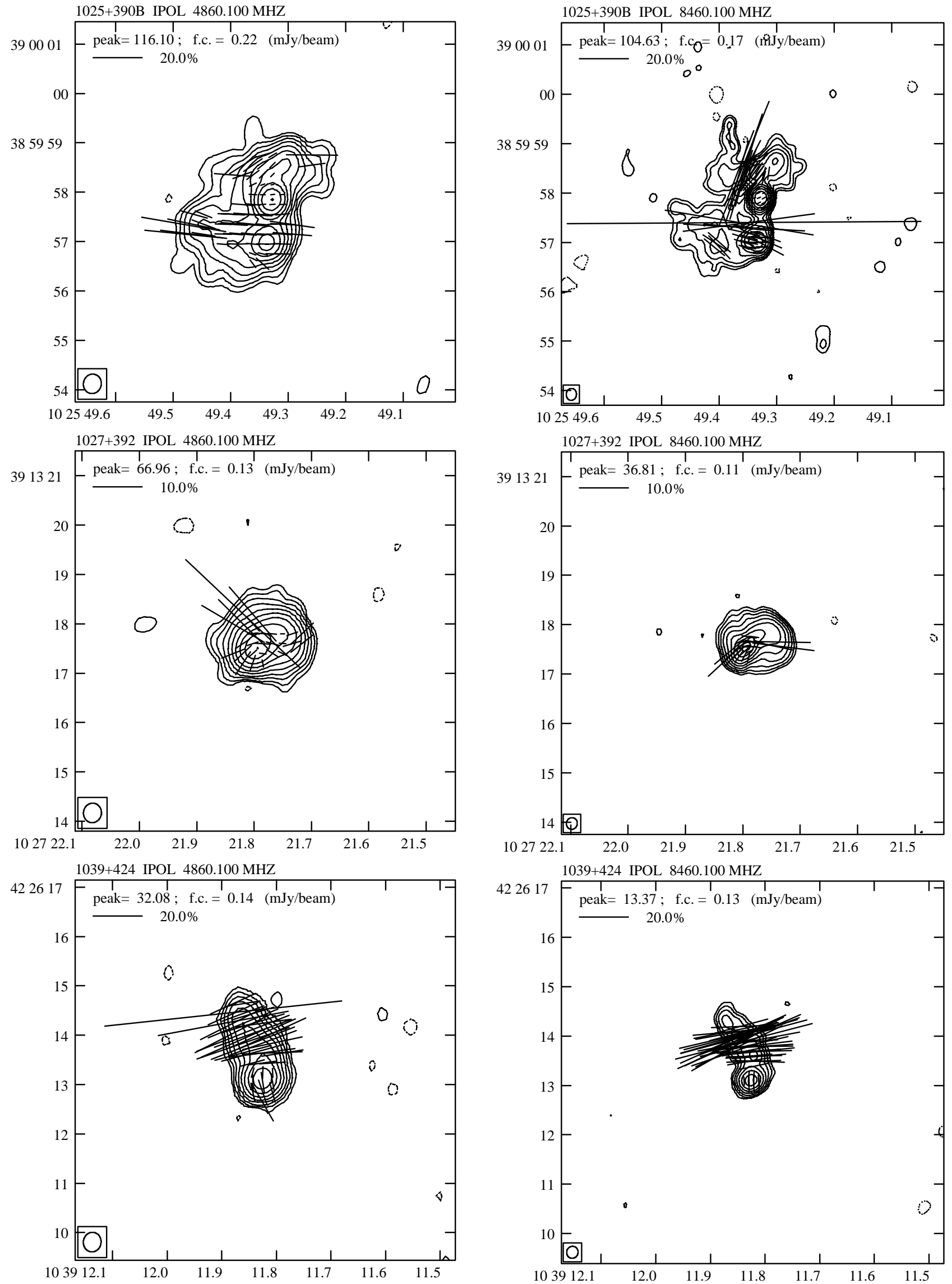

Fig. 3. continued 

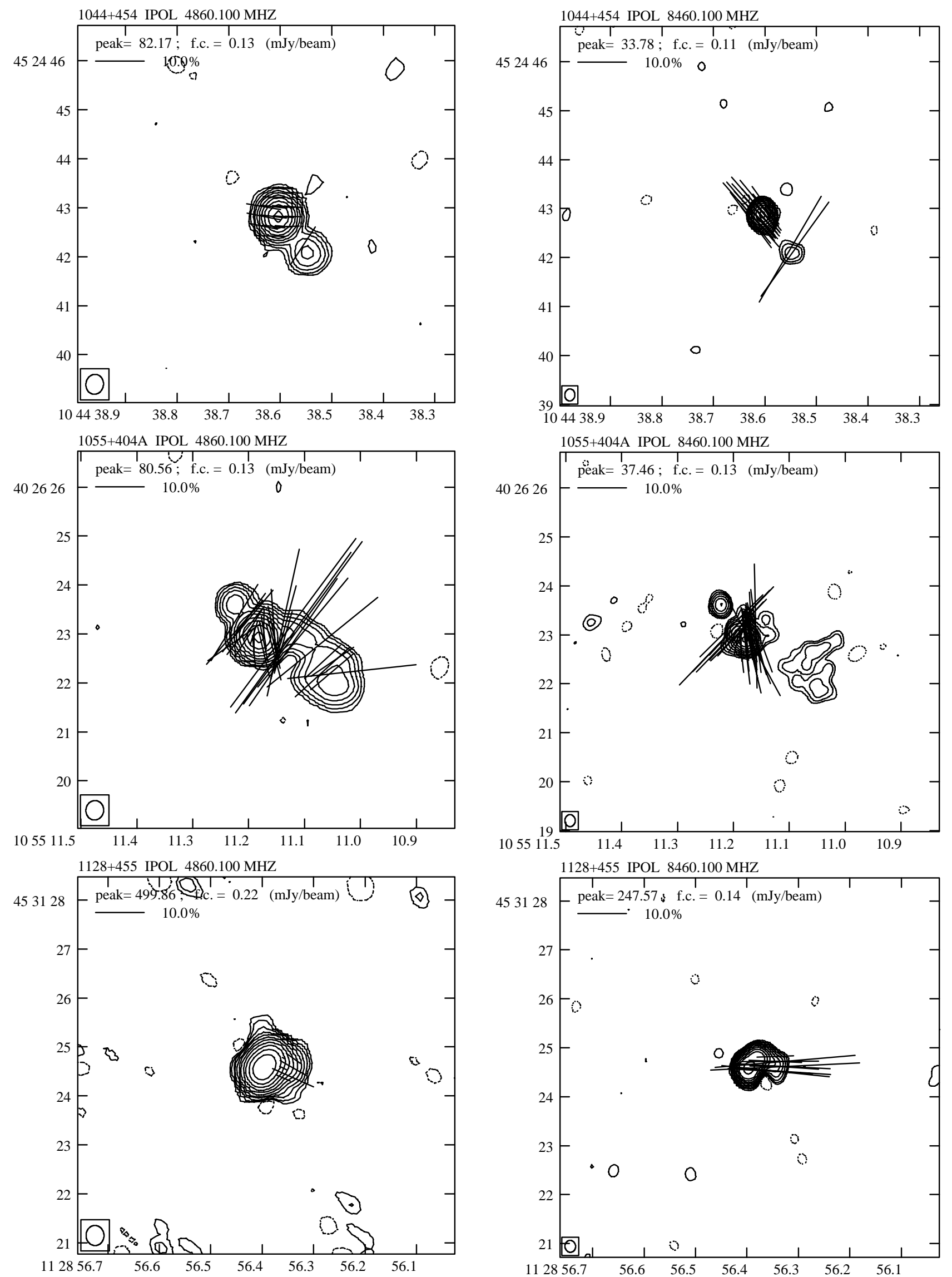

Fig. 3. continued 

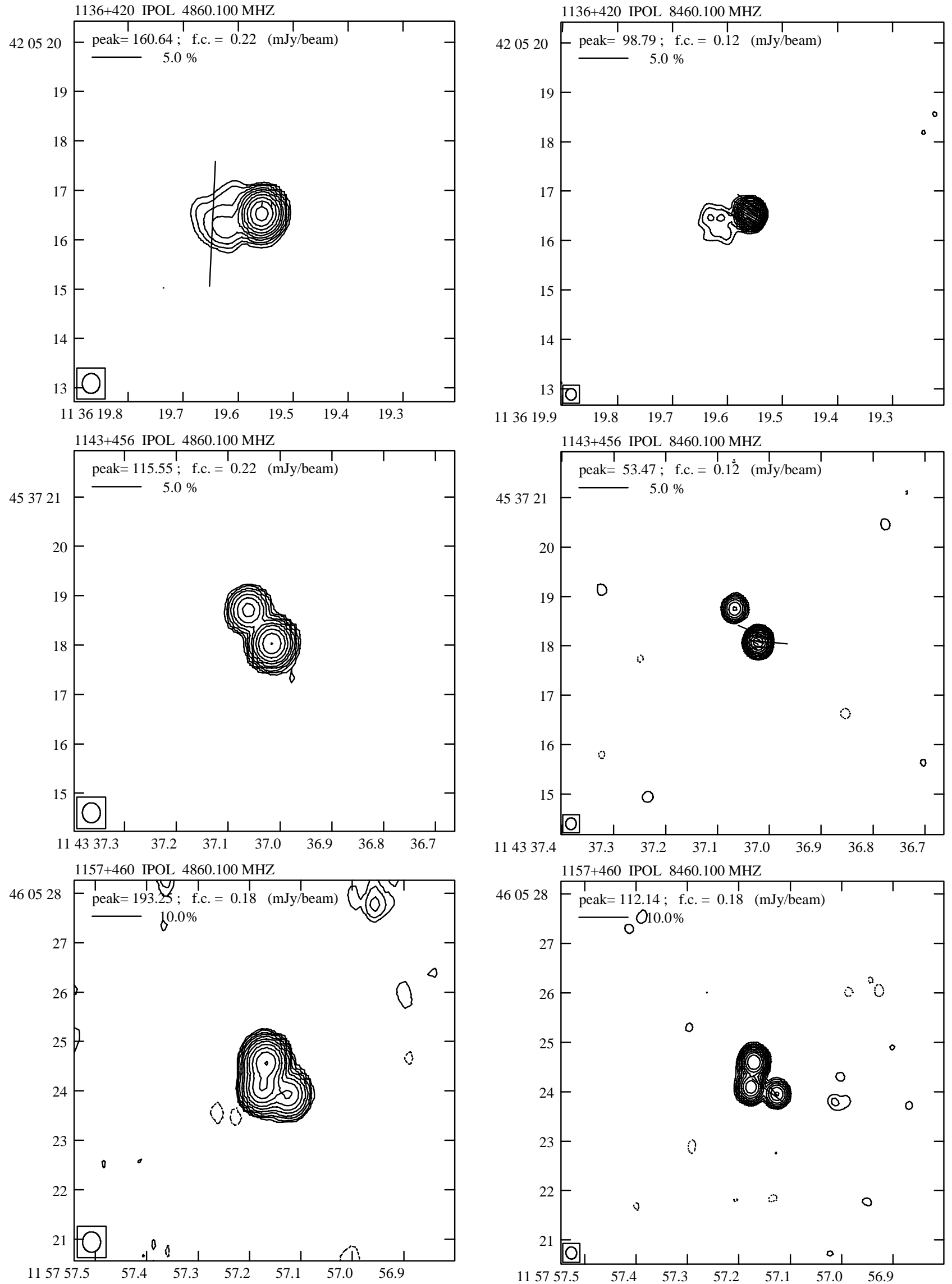

Fig. 3. continued 

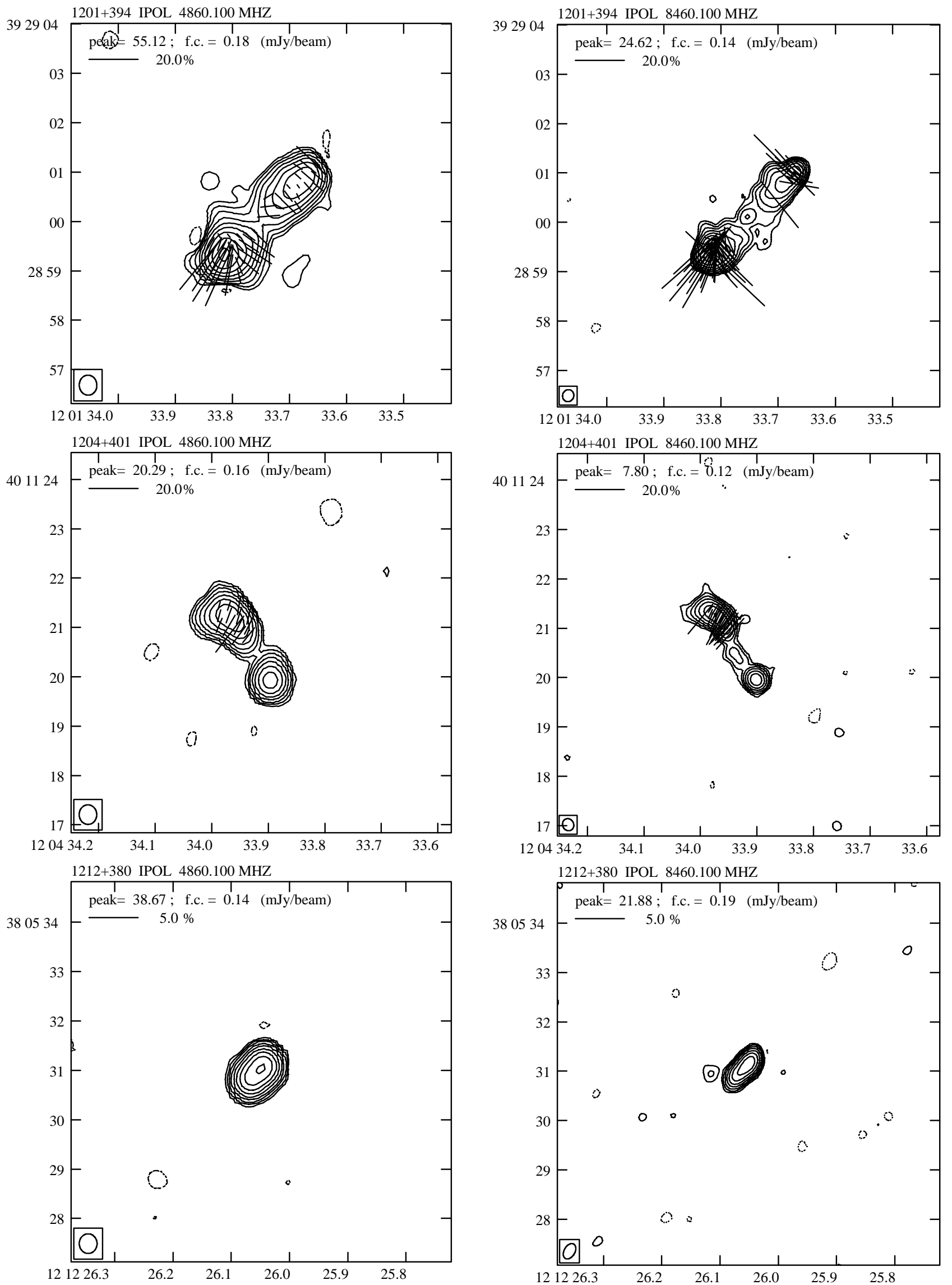

Fig. 3. continued 

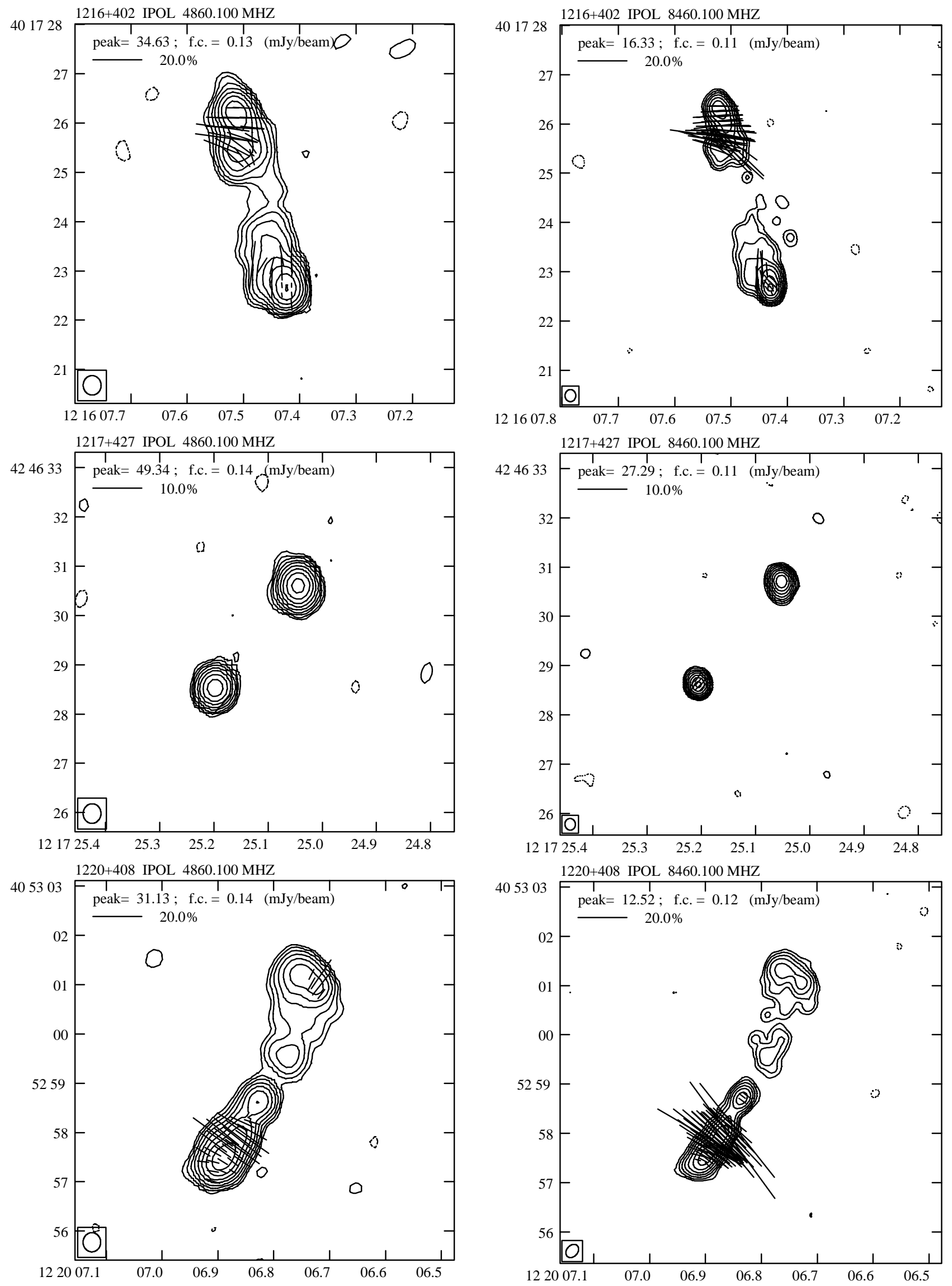

Fig. 3. continued 

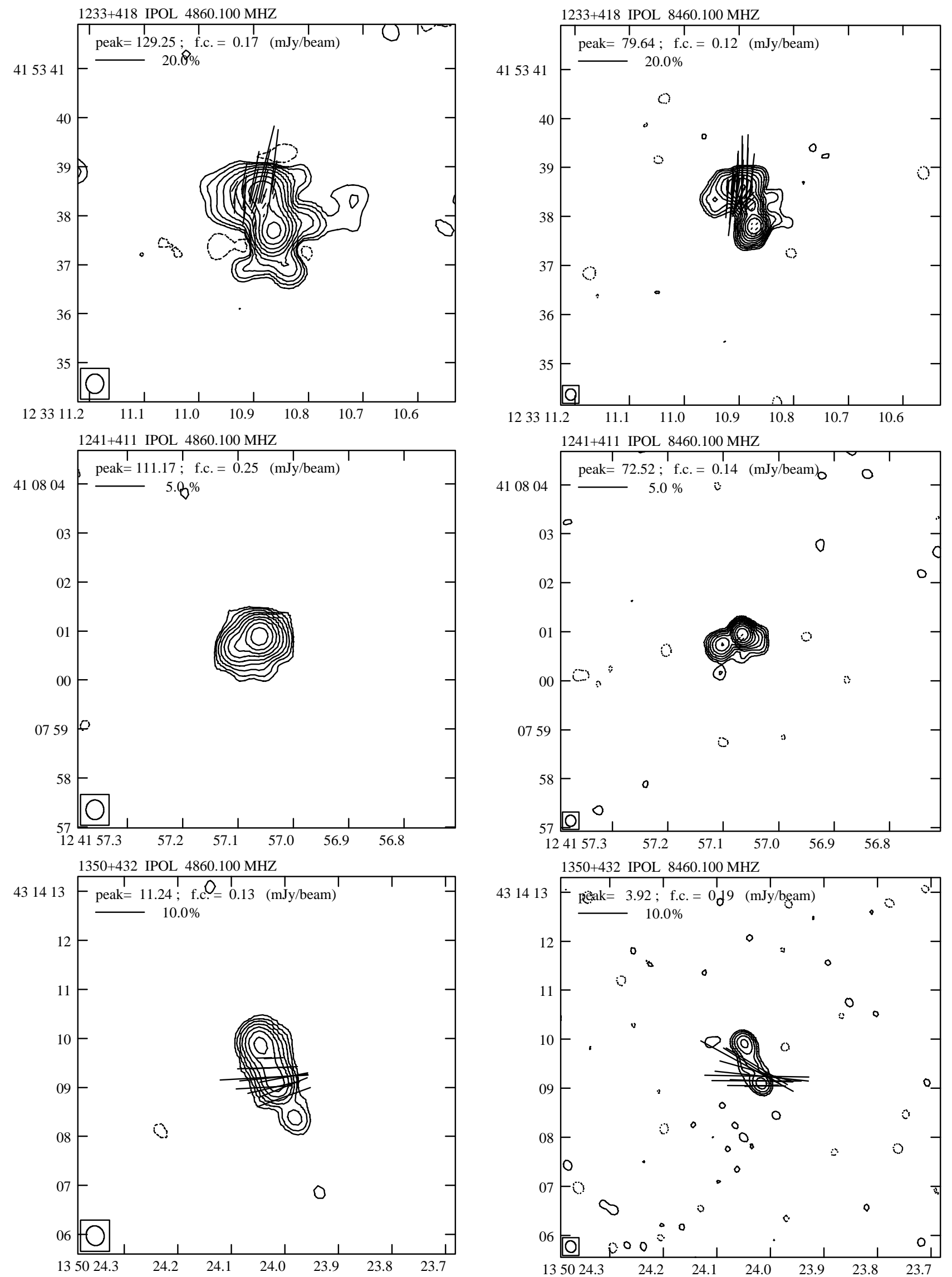

Fig. 3. continued 

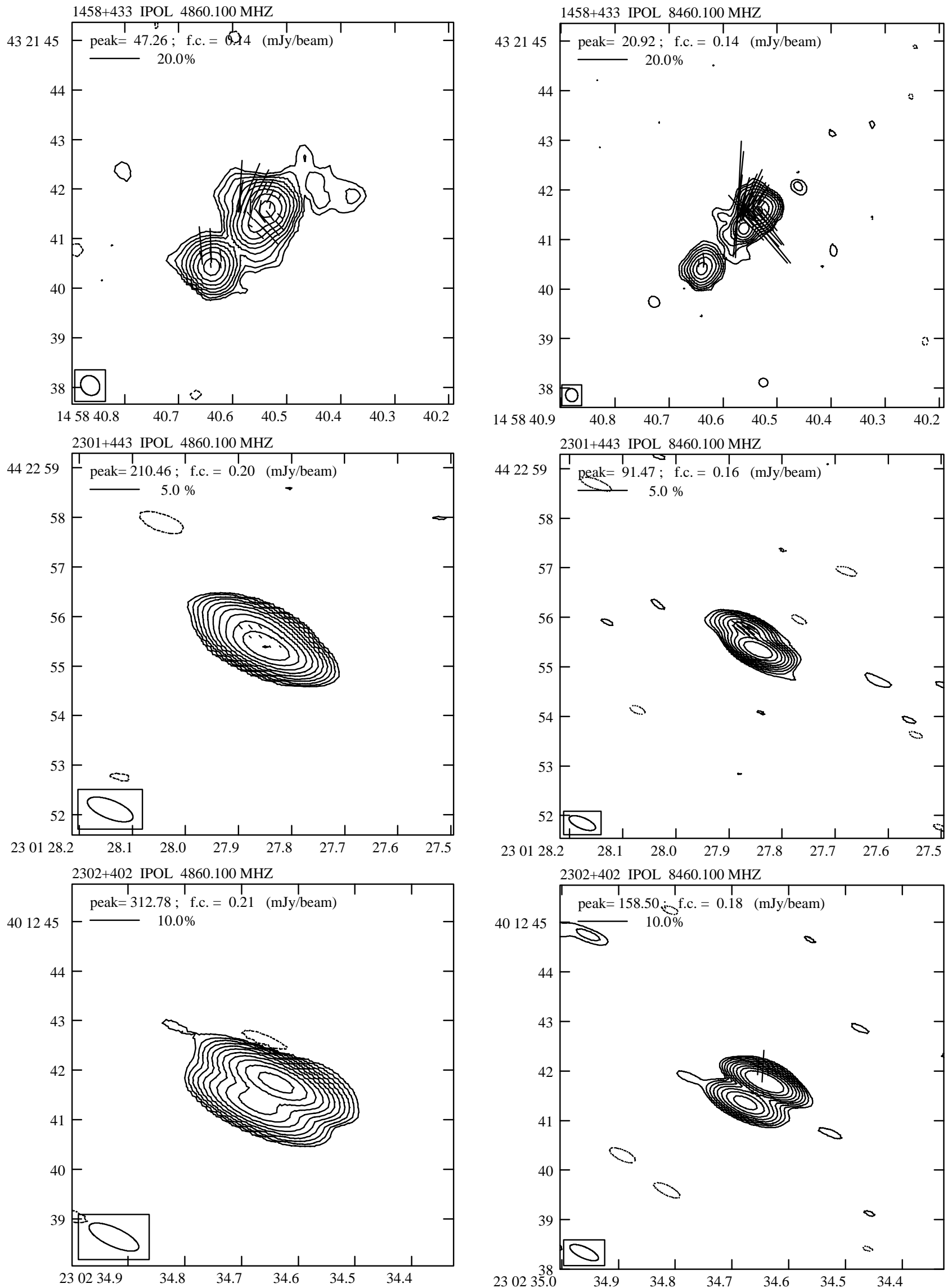

Fig. 3. continued 

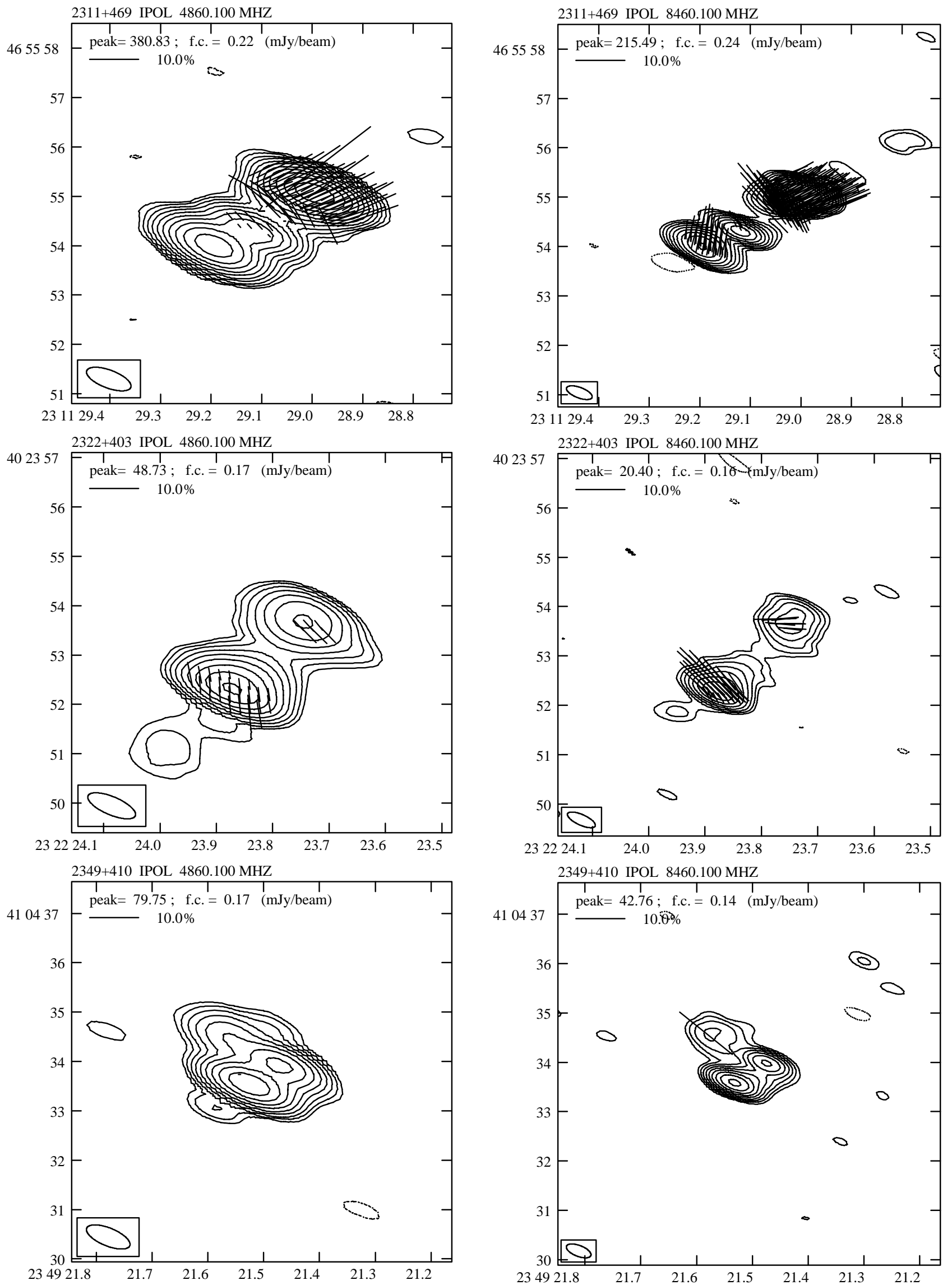

Fig. 3. continued 
- 0137+401: About 10 mJy (10\% and 16\% respectively) are missing in our VLA images at both 4.9 and $8.5 \mathrm{GHz}$ as compared with the values derived from the literature. The spectrum of the central component of this $T$ source $\left(\alpha_{4.9}^{8.5} \approx 0.74\right)$ is flatter than those of the outer components. A large variation of the position angle of the $E$ vector is seen within the northernmost component at $4.9 \mathrm{GHz}$.

$-0140+387$ : A large depolarization is seen in the southern component.

- 0144+432: In an 8.5 GHz image at the same resolution of the $4.9 \mathrm{GHz}$ (not shown) the bridge connecting the outer components is clearly visible. About $7 \mathrm{mJy}$ (10\%) are missing in our $8.5 \mathrm{GHz}$ VLA image, as compared with the values derived from the literature. The Faraday Rotation in the northern component is remarkably small.

- 0213+412: The spectrum of the central component of this $T$ source $\left(\alpha_{4.9}^{8.5} \approx 0.55\right)$ is much flatter than those of the outer components. Large variations of the position angle of the $\boldsymbol{E}$ vector are seen within components.

$-0222+422 \mathrm{~A}$ : The weak component at $\mathrm{RA}=22.69^{\mathrm{s}}$ and $\mathrm{DEC}=09.9^{\prime \prime}$, based on its location, is a candidate for the source core. However its spectral index is too uncertain to confirm this.

- 0228+409A: In between the two major components a $\sim 1$ mJy feature is clearly visible at $4.9 \mathrm{GHz}$, but it does not coincide with neither of the "blobs" seen at $8.5 \mathrm{GHz}$. About $10 \mathrm{mJy}$ (9\% and $17 \%$ respectively) are missing in our VLA images at both 4.9 and $8.5 \mathrm{GHz}$ as compared with the values derived from the literature. Large variations of the position angle of the $\boldsymbol{E}$ vector are seen within components.

$-0254+406$ : The weak component at $\mathrm{RA}=36.59^{\mathrm{s}}$ and $\mathrm{DEC}=33.1^{\prime \prime}$, based on its location is a candidate for the source core. However its spectral index is too uncertain to confirm this. The Faraday Rotation in the northern lobe is very small.

$-0255+460$ : This is one of the few resolved sources with no detectable polarization.

- 0701+392: A large asymmetry in fractional polarization between components is observed at both frequencies.

$-0722+393 \mathrm{~A}$ : This is one of the few resolved sources with no detectable polarization.

- 0729+437: Small Faraday Rotation is seen in both lobes.

- 0744+464: The spectrum of the central component of this $T$ source $\left(\alpha_{4.9}^{8.5} \approx 0.57\right)$ is much flatter than those of the outer components. There is a large asymmetry in fractional polarization between components at $8.5 \mathrm{GHz}$; the westernmost one shows strong depolarization and Faraday Rotation.
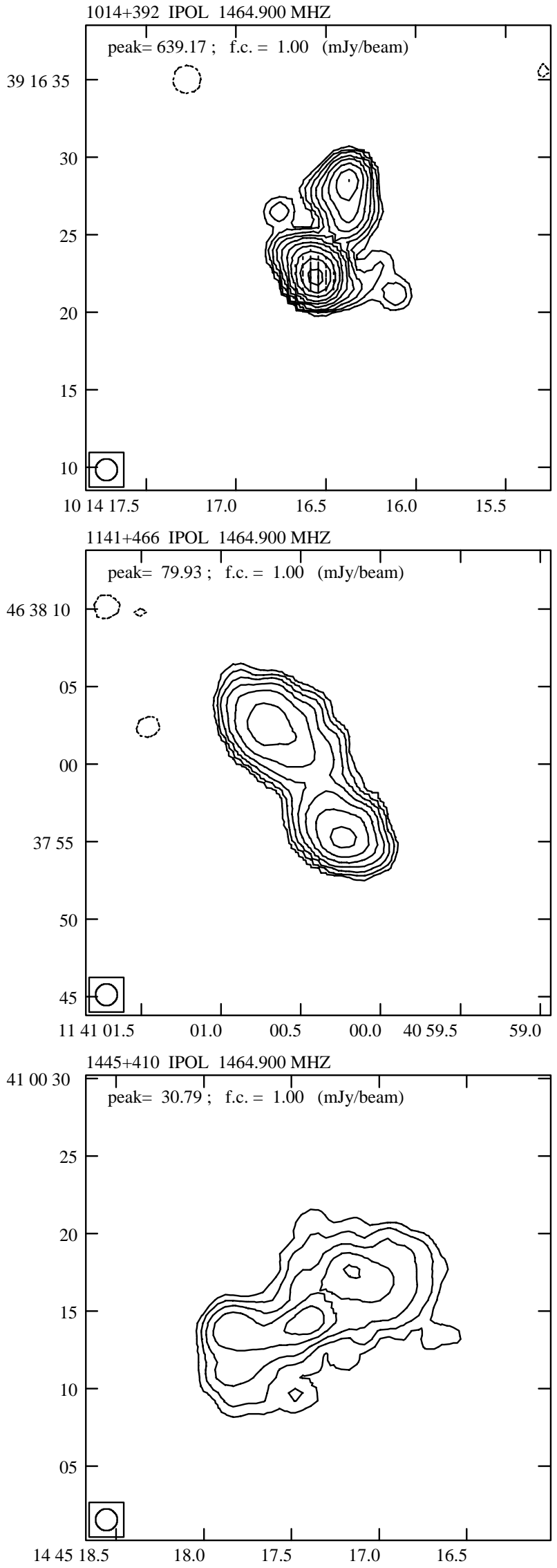

Fig. 4. Images of the radio sources observed at $1.5 \mathrm{GHz}$ only 
- 0748+413B: A weak radio source at $\mathrm{RA}=54.59^{\mathrm{s}}$ and Dec $=25.7^{\prime \prime},\left(13.8^{\prime \prime}\right.$ distant in PA $\left.114^{\circ}\right)$ with $S_{8.5}=$ $2.7 \mathrm{mJy}, S_{4.9}=4.6 \mathrm{mJy}$ and $S_{1.5}=17.3 \mathrm{mJy}$ is likely an unrelated source. About $30 \mathrm{mJy}(40 \%)$ and $10 \mathrm{mJy}$ $(30 \%)$ are missing in our 4.9 and $8.5 \mathrm{GHz}$ VLA images, as compared with the values derived from the literature. There is no obvious explanation for this. A large variation of the position angle of the $\boldsymbol{E}$ vector is seen across the structure.

- 0754+396: A weak radio source at $\mathrm{RA}=46.34^{\mathrm{s}}$ and Dec $=26.6^{\prime \prime},\left(11.1^{\prime \prime}\right.$ distant in PA $\left.-142^{\circ}\right)$, with $S_{8.5}<$ $0.1 \mathrm{mJy}, S_{4.9}=0.9 \mathrm{mJy}$ and $S_{1.5}=5.5 \mathrm{mJy}$ is likely an unrelated source. The Faraday Rotation is remarkably small across the whole source and the magnetic field appears very ordered.

$-0800+472$ : The western tail, seen in the $8.5 \mathrm{GHz}$ image, is not resolved by the $4.9 \mathrm{GHz}$ beam. Large changes in the position angle of the $\boldsymbol{E}$ vector are found within the main component at $8.5 \mathrm{GHz}$.

- 0805+406: This complicated object has been classified as a $T$ ? on the hypothesis that the westernmost extended structure is an unrelated source. The component at $\mathrm{RA}=40.71^{\mathrm{s}}$ and $\mathrm{DEC}=44.8^{\prime \prime}$, however, has a flattish spectrum, so that the object would rather be an $f c J$. If instead this is a single source, its total size becomes $\sim 3.7^{\prime \prime}$ or $\left[\sim 15.9 h^{-1} \mathrm{kpc}\right]$, i.e. still in the range for a CSS. Large changes in the position angle of the $\boldsymbol{E}$ vector are found within components but small Faraday Rotation.

- 0809+404: The eastern component shows little Faraday Rotation but is strongly depolarized.

$-0810+460 \mathrm{~B}$ : Large asymmetry in fractional polarization is found between components at $8.5 \mathrm{GHz}$. The southwestern one is strongly depolarized.

- 0814+441: The component at $\mathrm{RA}=8.04^{\mathrm{s}}$ and $\mathrm{DEC}=$ $54.4^{\prime \prime}$ has a flat spectrum and therefore it is the core. About $15 \mathrm{mJy}(18 \%)$ and $5 \mathrm{mJy}(10 \%)$ are missing in our 4.9 and $8.5 \mathrm{GHz}$ VLA images, as compared with the values derived from the literature.

- 0856+406: One of the few resolved sources which has no detectable polarization.

$-0930+389$ : The component at $\mathrm{RA}=0.78^{\mathrm{s}}$ and $\mathrm{DEC}=$ $9.7^{\prime \prime}$ has a flat spectrum and therefore it is the core. There is a large difference in the $\boldsymbol{E}$ vector position angle of the two components at $8.5 \mathrm{GHz}$. The southern component shows a large Faraday Rotation.

- 0951+422: The spectrum of the central component of this $T$ source $\left(\alpha_{4.9}^{8.5} \approx 0.5\right)$ is flatter than those of the outer ones. Large variations of the position angle of the $\boldsymbol{E}$ vector are seen within the easternmost component at both frequencies. The central and the southern components are largely depolarized.

The optical counterpart of this source, at the limit of the POSS red print, was classified as a galaxy, by
Vigotti et al. (1989), purely on morphological basis and its redshift estimated to be $z=0.28$. The morphological classification was also supported by an $R$ band CCD image from the $1.5 \mathrm{~m}$ Loiano (Bologna) telescope. However Falco et al. (1998), in their redshift survey of flat spectrum radio sources, identify the source with a quasar of $I=19.3$. Very likely the quasar is the object classified as a galaxy by Vigotti et al. (1989). An obscure point, leaving some residual doubts on the new identification, is that this source does not belong to the JVAS source list (Patnaik et al. 1992) from which Falco et al. (1998) state that they have selected their sources. Furthermore the source does not have a flat spectrum, like JVAS sources, and in any case it is fainter, at $5 \mathrm{GHz}$, than the selection flux density limit for their sample in which $0951+422$ is listed. Do we speak of the same source?

0955+390: The component at $\mathrm{RA}=42.99^{\mathrm{s}}$ and $\mathrm{DEC}=$ $43.5^{\prime \prime}$ has an inverted spectrum and therefore it is the core. There is a large variation of the position angle of the $\boldsymbol{E}$ vector within the eastern component but small average Faraday Rotation.

- 1025+390B: Extended structure surrounds the main components. The component at $\mathrm{RA}=49.33^{\mathrm{s}}$ and DEC $=57.9^{\prime \prime}$ has a flat spectrum and therefore it is the core. The integrated spectrum of the source, derived from the database by Vigotti et al. (1999) and the present data at $8.5, \mathrm{GHz}$ shows a significant flattening above this frequency, implying that the core dominates at 10.6 GHz.

$-1027+392$ : A sort of bent jet emerges from the compact structure, clearly seen at $8.5 \mathrm{GHz}$. Any fit with one or more components does not allow a proper source decomposition. We keep the decomposition into two substructures since this corresponds to what seen in polarization images, but note that the estimates of the individual spectra are uncertain.

- 1039+424: The extended feature appears as a knotty bent jet at $8.5 \mathrm{GHz}$; the component decomposition is not accurate at $1.5 \mathrm{GHz}$. A weak source at $\mathrm{RA}=12.08^{\mathrm{s}}$ and $\mathrm{DEC}=28.3^{\prime \prime}\left(15.5^{\prime \prime}\right.$ distant in $\left.\mathrm{PA} 10^{\circ}\right)$ with $S_{8.5}=$ $0.5 \mathrm{mJy}, S_{4.9}=1.4 \mathrm{mJy}$, and $S_{1.5}=5.9 \mathrm{mJy}$ is probably an unrelated source, although it is curious that the "jet" points approximately in its direction. A check made on the FIRST image (Becker et al. 1995) does not reveal any significant connection between the two. The small Faraday Rotation allows to see easily the change in the orientation of the magnetic field, from transverse to parallel to the jet.

- 1044+454: There is a large difference in the $\boldsymbol{E}$ vector position angle of the two components and large Faraday Rotation in the northern one. 

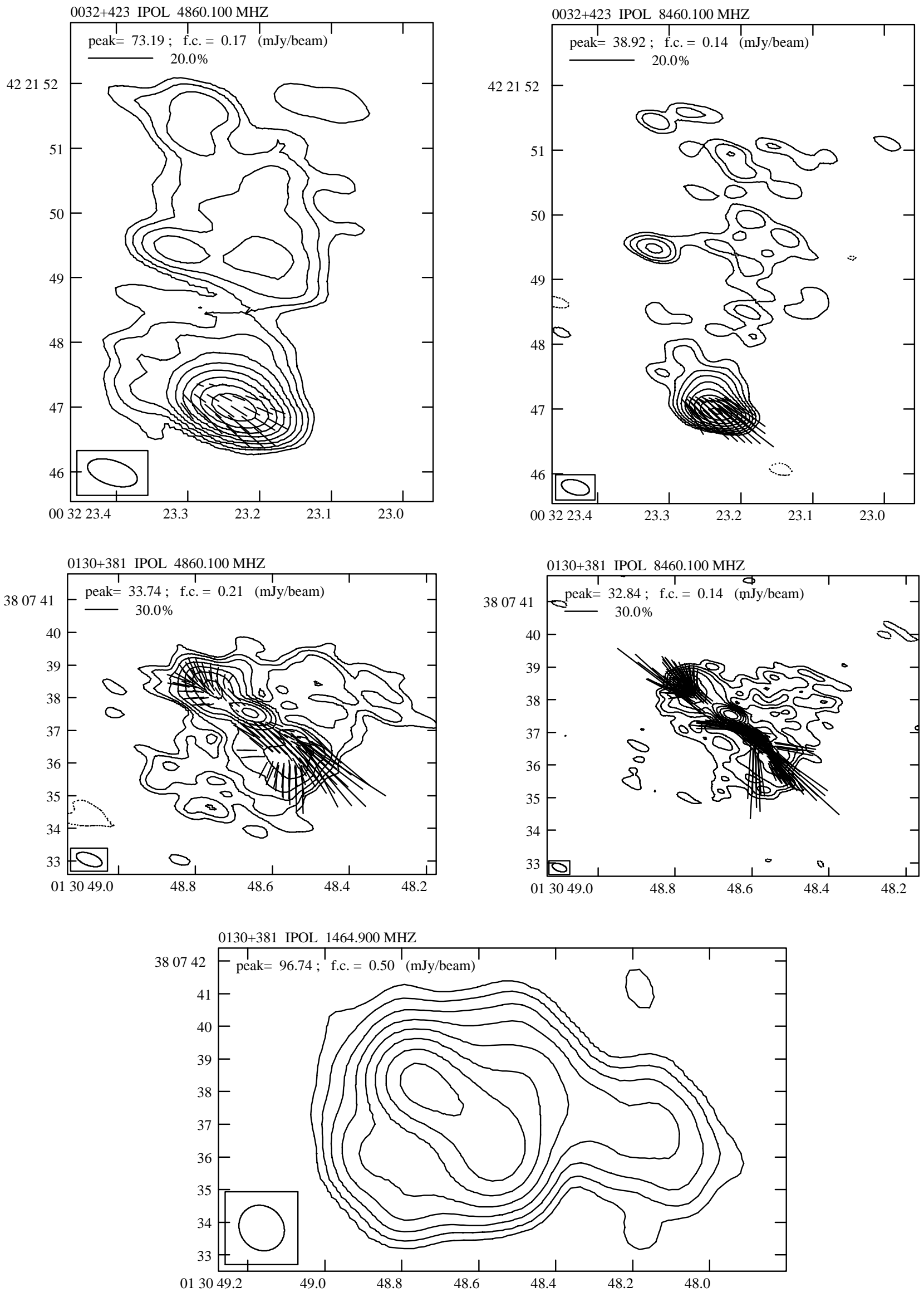

Fig. 5. Images of B3-VLA non-CSS sources at 4.9 (left) and 8.5 (right) GHz; for $0130+381$ the $1.5 \mathrm{GHz}$ image is also given 

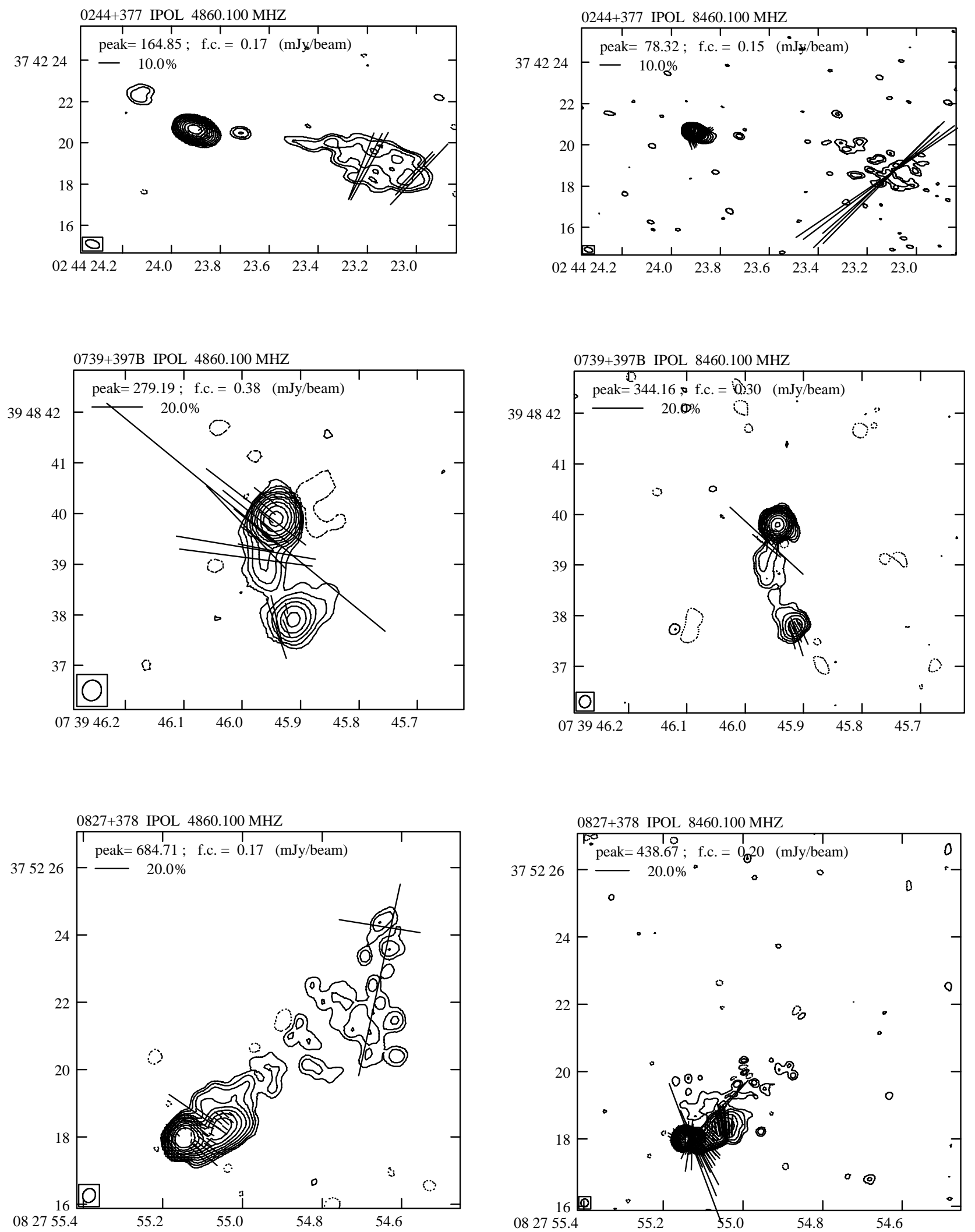

Fig. 5. Continued. Images of B3-VLA non-CSS sources at 4.9 (left) and 8.5 (right) $\mathrm{GHz}$ 

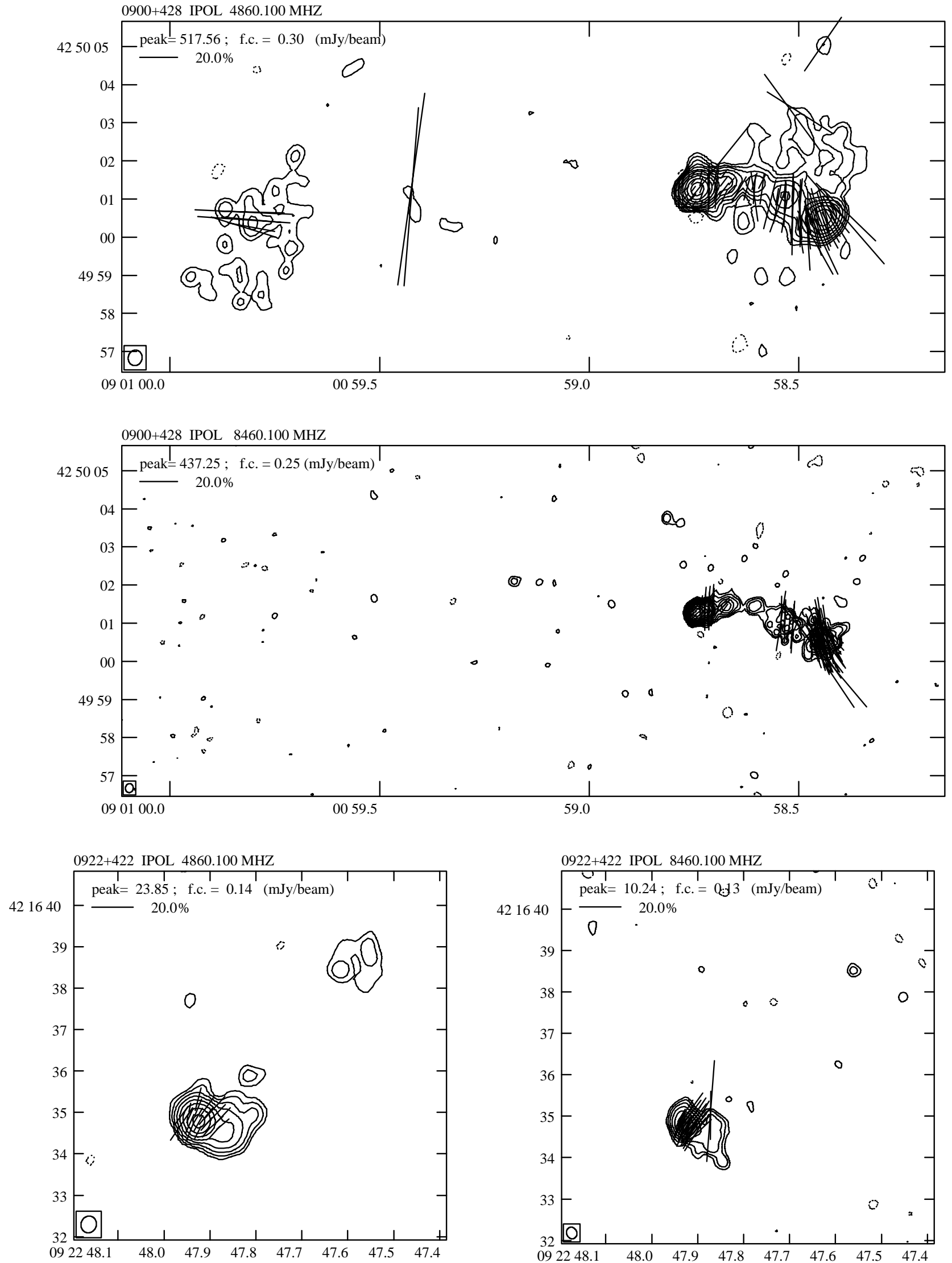

Fig. 5. Continued. Images of B3-VLA non-CSS sources at 4.9 (top \& left) and 8.5 (bottom $\&$ right) $\mathrm{GHz}$ 

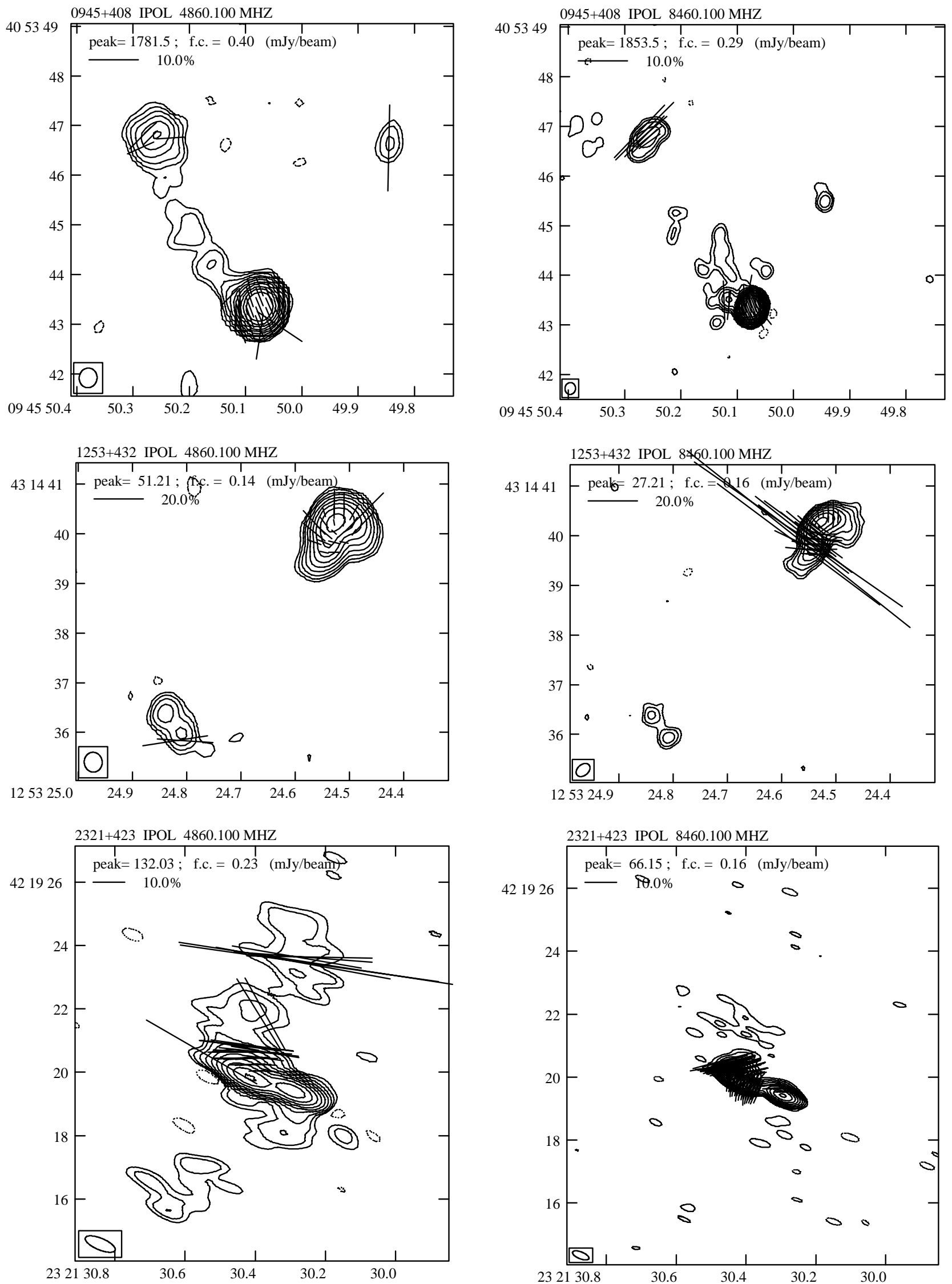

Fig. 5. Continued. Images of B3-VLA non-CSS sources at 4.9 (left) and 8.5 (right) $\mathrm{GHz}$ 
C. Fanti et al.: B3-VLA CSSs
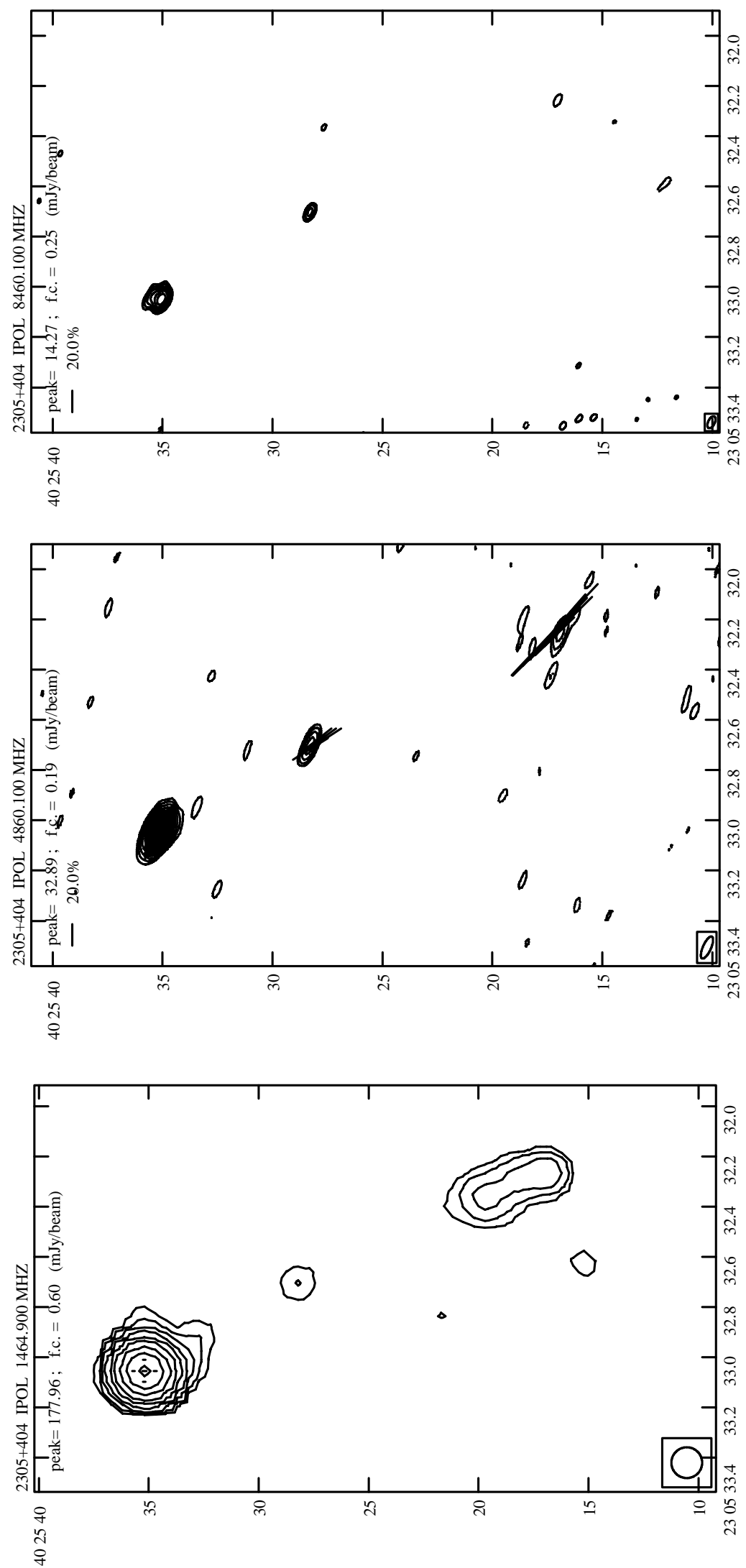

Fig. 5. Continued. Images of 2305+404 at 1.5 (left), 4.9 (middle) and 8.5 (right) $\mathrm{GHz}$ 
$-1055+404 \mathrm{~A}:$ The component at $\mathrm{RA}=11.22^{\mathrm{s}}$ and DEC $=23.6^{\prime \prime}$ shows a flat spectrum and therefore it is the core. A large variation of the position angle of the $\boldsymbol{E}$ vector is seen within the central component.

- 1133+432: This $U$ source (no image shown) has a spectrum peaking at $\approx 1 \mathrm{GHz}$, therefore it is a genuine GPS.

$-1143+456$ : There is very little polarization at $8.5 \mathrm{GHz}$ and in the southern component only.

$-1157+460$ : We classified this object as a $T$ ? (see Table 1 ) but it could be a more complex object. No significant polarized emission is present.

- 1201+394: Large variations of the position angle of the $\boldsymbol{E}$ vector are seen within the south-eastern component. There is little Faraday Rotation in both components.

- 1204+401: The northern component is much more polarized than the southern one at both frequencies.

$-1212+380$ : This is one of the few resolved sources with no detectable polarization.

- 1216+402: There is little Faraday Rotation in the southern component.

- 1220+408: This "blobby" structure is more likely to be an asymmetric double source, rather than a jet, since there is no indication, in the present data, of a core component. The southern component is significantly more polarized than the northern one at both frequencies and shows little Faraday Rotation.

- 1233+418: Polarized emission is detected only in the northern component at both frequencies; the Faraday Rotation is very small.

- 1225+442: About $13 \mathrm{mJy}$ and $5 \mathrm{mJy}$ (12\% and $11 \%$ respectively) are missing in our 4.9 and $8.5 \mathrm{GHz}$ VLA images, as compared with the values derived from the literature.

$-1241+411$ : This is one of the few resolved sources with no detectable polarization.

$-1343+386$ : In the field of this $U$ source (no image shown) an object, at $\mathrm{RA}=26.59^{\mathrm{s}}, \mathrm{DEC}=24.8^{\prime \prime}\left(12.9^{\prime \prime}\right.$ distant in PA $0^{\circ}$ ) with $S_{8.5}=7.5 \mathrm{mJy}, S_{4.9}=14.2 \mathrm{mJy}$ and $S_{1.5}=41.3 \mathrm{mJy}$ is presumably a field object.

$-1350+432$ : It is classified as a $D$ ? in Table 1 although it could have a more complicated structure. Its polarization is quite asymmetric.

- 1432+428B: This $U$ source (no image shown) has a spectrum peaking at $\approx 1 \mathrm{GHz}$, therefore it is a genuine GPS.

- 1458+433: The spectrum of the central component of this $T$ source $\left(\alpha_{4.9}^{8.5} \approx 0.55\right)$ is flatter than those of the outer components. There are large variations of the position angle of the $\boldsymbol{E}$ vector within the northwestern component but little average Faraday Rotation in this and in the central component.
$-2302+402$ : This is one of the few resolved sources with no detectable polarization.

$-2311+469:$ The component at RA $=29.12^{\mathrm{s}}$ and DEC $=54.3^{\prime \prime}$ has a flattish spectrum and is likely to be the core. The north-western weak component, at RA $28.8^{\mathrm{s}}$, DEC 56.0" , although present at both frequencies is likely to be an artifact. The south-eastern component is significantly more depolarized than the rest of the radio source. Large variations of the position angle of the $\boldsymbol{E}$ vector are found within the north-western component.

$-2322+403:$ The south-eastern weak structure visible at $4.9 \mathrm{GHz}$, is totally resolved at $8.5 \mathrm{GHz}$.

- 2349+410: No significant polarized emission is present in this peculiar $T$ source.

\section{Sources from Table 2}

- 0130+381: This object resembles a typical FRI radio source, with a strong core and prominent jets. The core is unpolarized while the jets display high percentage polarization, with the $\boldsymbol{E}$ vector running mostly parallel to their axis. The Faraday Rotation is remarkably small.

$-0244+377$ : The component at $\mathrm{RA}=23.72^{\mathrm{s}}$ and $\mathrm{DEC}=$ $20.4^{\prime \prime}$, based on its location, is a candidate for the source core. However its spectral index is too uncertain to confirm this. Large variations of the position angle of the $\boldsymbol{E}$ vector are seen within components.

0739+397B: The component at $\mathrm{RA}=45.94^{\mathrm{s}}$ and $\mathrm{DEC}$ $=39.8^{\prime \prime}$ has a spectrum rising at higher frequencies and therefore it harbors the core. The polarized signal of the extended component traces the beginning of the $\mathrm{N}-\mathrm{S}$ jet.

- 0827+378: The decomposition of the easternmost component into two structures is not very reliable at $4.9 \mathrm{GHz}$, due to the low resolution, so that the apparently flat spectrum of component at $\mathrm{RA}=55.12^{\mathrm{s}}$ and DEC $=17.9^{\prime \prime}$ is very uncertain. The bright component, as a whole, has however a flattish spectrum $\left(\alpha_{4.9}^{8.5} \approx 0.5\right)$.

- 0900+428: About $260 \mathrm{mJy}(38 \%)$ and $350 \mathrm{mJy}(38 \%)$ are missing in the 4.9 and $1.5 \mathrm{GHz}$ images, as compared with the values derived from the literature. This is mostly due to the extended features surrounding the western component which are much too resolved by our observations, as proved by the comparison of our image at $1.5 \mathrm{GHz}$ and the FIRST one. The easternmost extended feature is totally undetetected at $8.5 \mathrm{GHz}$, and this is consistent with the signal expected at this frequency from the literature values.

$-0922+422$ : A jet emerges from the brightest component and bends towards north. The north-western component is barely visible at $8.5 \mathrm{GHz}$ and its flux density 
and position at this frequency given in Table 3 bis refer to the weak "hot spot" only. The spectral index is computed between 5 and $1.5 \mathrm{GHz}$. About 15 (23\%) and 5 (17\%) mJy are missing in our 4.9 and $8.5 \mathrm{GHz}$ VLA images, as compared with the values derived from the literature.

- 2305+404: Very asymmetric triple source; the central component at $\mathrm{RA}=32.70^{\mathrm{s}}$ and $\mathrm{DEC}=28.3^{\prime \prime}$ has a flat spectrum and therefore it is the source core.

- 2321+423: $S$-shaped structure with low surface brightness "wings", perpendicular to the source main structure.

\section{Discussion}

Since forthcoming papers will deal in detail with the properties of this sample, the discussion presented here is intentionally very schematic, a sort of guided interpretation of the data reported in the figures and tables. Here we intend to simply summarize the basic sample characteristics as derived from the present "low" resolution observations.

\subsection{Radio morphology}

In the morphological classification given at the end of Table 1, we use both the low and the high resolution information available to us at present. In the definition of lobes and jets we mostly use a "low resolution" approach, while we define as (flat spectrum) "core" a "compact" component with a flat or inverted or convex spectrum. In a few cases, however a weak compact component, located in between the two extended lobes, is mentioned in the notes (Sect. 5.6) as the possible source core, although, because of the low signal-to-noise ratio, the criteria of the flat spectrum cannot be applied. Based on the definitions given in Table 1 we have that:

-Twenty nine objects $(\approx 33 \%)$ are unresolved $(U, 22)$ or slightly resolved $(S R, 7)$. No images are shown for the $U$ and for two of the $S R$ sources (Sect. 5.5).

-Forty nine objects $(\approx 56 \%)$ are Double $(D, 39)$ or Triple $(T, 10)$. In a about half of the $T$ sources (mentioned in the notes) the central component, although steep, has a spectrum flatter than the outer components. It is likely an almost unresolved jet (plus possibly a flat spectrum core). -One object is complex $(C x)$ and one an $f c J$ (flat core + Jet).

-Seven $(\approx 8 \%)$ objects are steep core + Jet $(s c J)$. We suspect that some of them could be $f c J$ sources where a weak flat spectrum core is confused with brighter steep spectrum emission from the jet.

Only in a minority of sources $(\lesssim 10 \%)$ we detect or possibly detect a flat spectrum radio core. The scarcity of core detections is very likely due to insufficient angular resolution with respect to the overall radio size.

For what we can say so far, the morphologies of quasars are not different from those of galaxies+ empty fields.

\subsection{Spectral indices}

The integrated source spectra show a clear steepening with frequency, as seen from the average spectral indices in the frequency ranges $8.5-4.9 \mathrm{GHz}, 4.9-1.4 \mathrm{GHz}, 1.4-0.4 \mathrm{GHz}$. For this analysis we used our own flux densities except for those at $1.4 \mathrm{GHz}$ which are from NVSS, as they are believed to be more accurate. We find:

$\begin{array}{ll}<\alpha_{4.9}^{8.5}>=1.06 \pm 0.03 & \sigma_{\alpha}=0.26 \\ <\alpha_{1.4}^{4.9}>=0.97 \pm 0.02 & \sigma_{\alpha}=0.21 \\ <\alpha_{0.4}^{1.4}>=0.71 \pm 0.03 & \sigma_{\alpha}=0.26 .\end{array}$

Typical errors on the spectral index of individual sources, due to noise and calibration uncertainties, are $\approx 0.05-0.07$ in each frequency range. Resolution effects may account at most for 0.04 of the spectral steepening between the two highest frequency ranges. In the low frequency interval 15 sources have $\alpha_{0.4}^{1.4} \leq 0.5$ and 13 of them have $L L S \leq$ $0.5 h^{-1} \mathrm{kpc}$ (out of 21 sources in this $L L S$ class). Such a low frequency flattening is likely due to synchrotron selfabsorption, as confirmed by compilation of the integrated spectra from the database by Vigotti et al. (1999). The spectra of two sources, $1133+432$ (see also Snellen 1995) and $1432+428 \mathrm{~B}$, peak at about $1 \mathrm{GHz}$, therefore these are genuine GPSs. If we recompute $<\alpha_{0.4}^{1.4}>$ excluding the 21 small size sources, we get $\left\langle\alpha_{0.4}^{1.4}\right\rangle=0.81 \pm 0.02 \quad \sigma_{\alpha}=$ 0.17

As seen from the above numbers, the integrated spectra show a considerable curvature from the lower to the higher frequencies. Defining a curvature parameter as $C=\alpha_{4.9}^{8.5}-$ $\alpha_{0.4}^{1.4}$ we find:

$<C>=0.28 \pm 0.02 \quad \sigma=0.17 \quad$ (the 21 small size sources excluded).

In Fig. 6 we show a plot of $C$ vs. $\alpha_{0.4}^{1.4}$ (for clarity we have not plotted $1133+432$ which has $\left\langle\alpha_{0.4}^{1.4}\right\rangle=-0.4$ and $C=1.4)$. About $21 \%(18 / 87)$ of the sources have $C>0.5$. Only one $(1025+390 \mathrm{~B}$, see notes to the sources $)$ shows a high frequency flattening $(C<0)$. As expected the $U$ sources ( + in figure) have mostly flat $\alpha_{0.4}^{1.4}$ and large $C$, consistent with the presence of synchrotron selfabsorption. The majority of extended sources ( $*$ in figure) show a milder curvature.

The high frequency steepening is clearly visible also in the spectra of the individual radio components. An analysis of the differences between the high frequency and the three-point spectral index of the source components (Table 3), in fact, shows that, on average, this difference is $0.09 \pm 0.01$.

We have also compared the high frequency spectral indices of the two (outermost) components in the $D$ (and $T$ ) sources. The absolute value of the spectral indices difference has a dispersion $\sigma_{\alpha} \approx 0.3$, which is not accounted for by the errors. If we compute the difference of the spectral index between the more luminous and the less luminous side of the radio source, we find that this difference has a median value of $-0.14 \pm 0.09$ (error is $2 \sigma$ ) implying that 


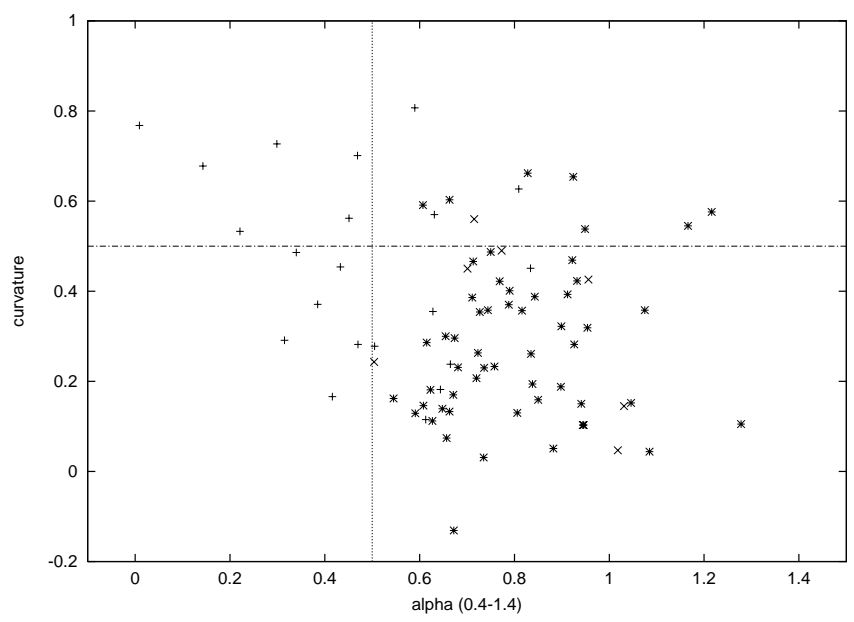

Fig. 6. Plot of $C$ vs. $\alpha_{0.4}^{1.4} \cdot(+)$ refer to the $U,(\times)$ to the $S R$ and $(*)$ to the extended sources

the more luminous side of the radio source has, on average, a flatter spectrum (Fig. 7). The distribution of the differences is slightly skewed and is still larger than expected from statistical errors.

No difference in the spectral properties is found between $T$ and $D$ (or $D c$ ) sources.

\subsection{Polarization properties}

Among the $U$ sources only $4 / 22$ are in fact polarized at either 4.9 or $8.5 \mathrm{GHz}$. On the contrary the majority of the resolved sources do show polarization at one or both frequencies: only $7 / 65$ do not show any detectable polarization (Sect. 5.6). Several show marked asymmetries in the two sides of the radio source (see notes in Sect. 5.6).

About $52 \%$ and $56 \%$ of the components are polarized at some level, at 4.9 and $8.5 \mathrm{GHz}$ respectively. These figures become $38 \%$ and $42 \%$ if we consider only the detections above $4 \%$. Furthermore as many as $\approx 18 \%$ and $\approx 21 \%$ of the source components have $m \geq 10 \%$ respectively at 4.9 and $8.5 \mathrm{GHz}$ (Figs. 8a,b). This is similar to what found by van Breugel et al. (1989) at 4.9 and $15 \mathrm{GHz}$, with VLA observations at resolutions similar to ours and by Akujor \& Garrington (1995) at $8.4 \mathrm{GHz}$. When upper limits are taken into account, the median values of percentage polarization, for the whole sample, are $m_{8.5} \approx 6_{-1}^{+2}$ and $m_{4.9} \approx 4_{-1}^{+2}$ (uncertainties are $2 \sigma$ ).

In radio components polarized at both frequencies we have computed the Faraday Rotation angle, $\Delta \chi$. This has a median absolute value of $\approx\left(16_{-7}^{+6}\right)^{\circ}$ (uncertainties are $2 \sigma)$, assuming no ambiguities ${ }^{4}$. In about $40 \%$ of cases $\Delta \chi<10^{\circ}$; the remaining $60 \%$ is spread more or less uniformly up to $\approx 45^{\circ}$, with a tail extending to $\approx 80^{\circ}$. In general the rotation angles in components of the same source are similar. Exceptions are mentioned in the notes (Sect. 5.6).

\footnotetext{
4 An ambiguity of $n \pi$ corresponds, in the observer frame, to an ambiguity of $(1350 \times n) \mathrm{rad} / \mathrm{m}^{2}$ in the Rotation Measure which, in general, seems unlikely.
}

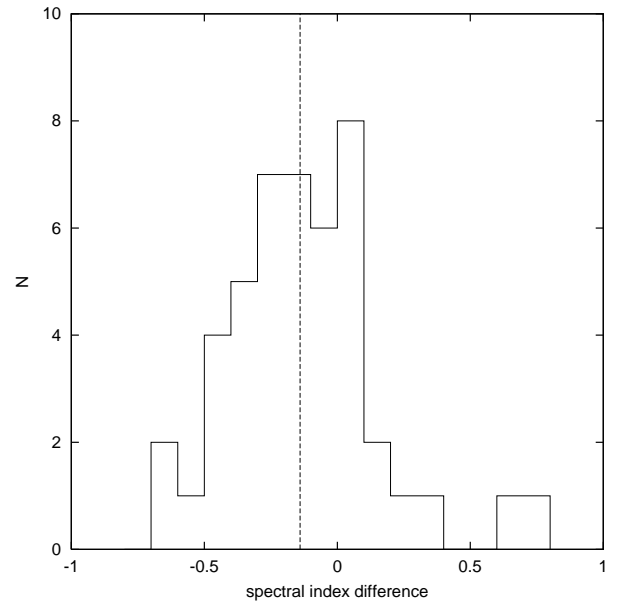

Fig. 7. Distribution of the differences between the high frequency spectral indices of the more luminous and the less luminous of the two (outermost) components in $D$ (and $T$ ) sources. The vertical line represents the median

We have also examined the depolarization ratio defined as $D P_{8.5}^{4.9}=m_{4.9} / m_{8.5}$. The distribution of these ratios peaks at $\approx 0.7$. About $12 \%$ of radio components appear to have an unusual $D P_{8.5}^{4.9} \geq 1.5$, not accounted for by the formal errors on $m$.

We did not find any correlation between $D P_{8.5}^{4.9}$ and Faraday Rotation angle.

\subsection{Linear sizes distribution}

The range we explored here goes from projected linear size $\approx 20 h^{-1}$ pc (limit of our selection) down to a few tenths of kpc, corresponding to our resolution limit. Sizes are evenly distributed in power, but we do not expect to see any trend, given the small explored size range.

The shape of the $L L S$ distribution function, is instead an important tool to study the evolution of powerful radio sources in the youth scenario (see Fanti et al. 1995; Readhead et al. 1996; O'Dea \& Baum 1997; Fanti 2000). In our sample this function can be fitted pretty well by the power law

$\frac{\mathrm{d} N(L L S)}{\mathrm{d}(L L S)} \propto(L L S)^{-m} \quad$ with $\quad m=0.6 \pm 0.1$

down to $L L S \approx 0.5 h^{-1} \mathrm{kpc}$. The exponent has the same value found by Fanti et al. (1995) using the $3 C R \& P W$ sample and does not confirm the flattening of the diameter counts for $L L S \lesssim 10 h^{-1} \mathrm{kpc}$ suggested by O'Dea \& Baum (1997). In order to increase the count statistics of the under-populated largest $L L S$ bins we plot in Fig. 9 the number-size relation in bins of equal $\log ($ size $)$. This converts the above relation into $\mathrm{d} N(L L S) / \mathrm{d}[\log (L L S)] \propto$ $(L L S)^{-m+1}$. Note that the uncertainty on size for the distant sources without $z$ is not likely to affect the distribution appreciably (see discussion in Sect. 3). Among our sources eighteen $(\sim 21 \%)$ of have $L L S \leq 0.4 h^{-1}$ and at the moment we cannot determine the $L L S$ distribution 

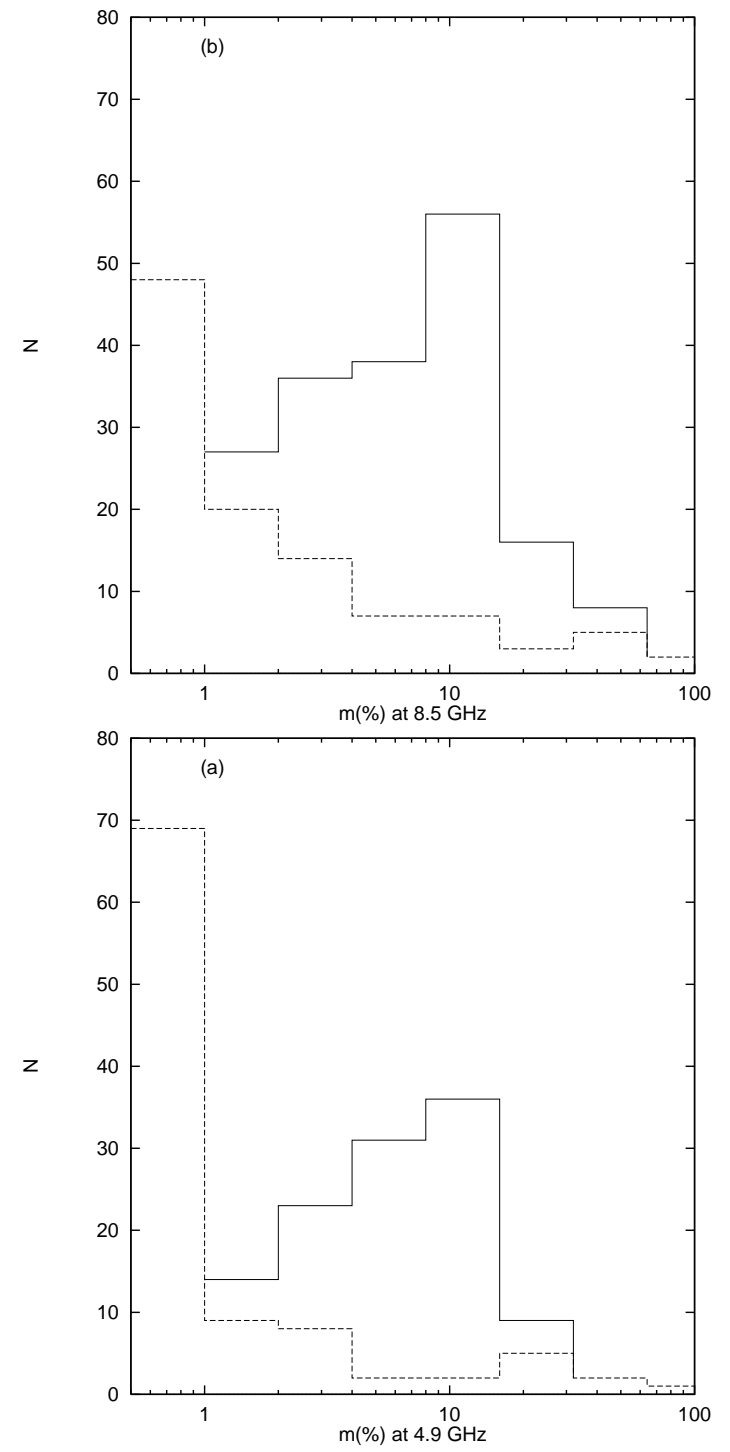

Fig. 8. Percentage polarization at 4.8 a) and 8.5 b) $\mathrm{GHz}$ for the source components. The dotted line represents upper limits, the continuous line the sum of upper limits and actual values in each bin

function below this value. This will be possible with the VLBI data which are being analyzed (Sect. 7). Their number, however, is consistent with the extrapolation to zero of the $\mathrm{d} N(L L S) / \mathrm{d}(L L S)$ relation found for the larger radio sources.

\section{Summary and future work}

a) We have presented a new complete sample of 87 CSS radio sources selected from a low frequency survey (B3 at $0.4 \mathrm{GHz}$ ) and studied at three frequencies (1.5, 4.9, $8.5 \mathrm{GHz}$ ) with the $V L A-A$.

b) Sixty two percent of the objects have a redshift either spectroscopic or photometric.

c) About $75 \%$ of the sources are more or less resolved at 8.5 GHz, with linear sizes ranging from $20 h^{-1} \mathrm{kpc}$ (upper

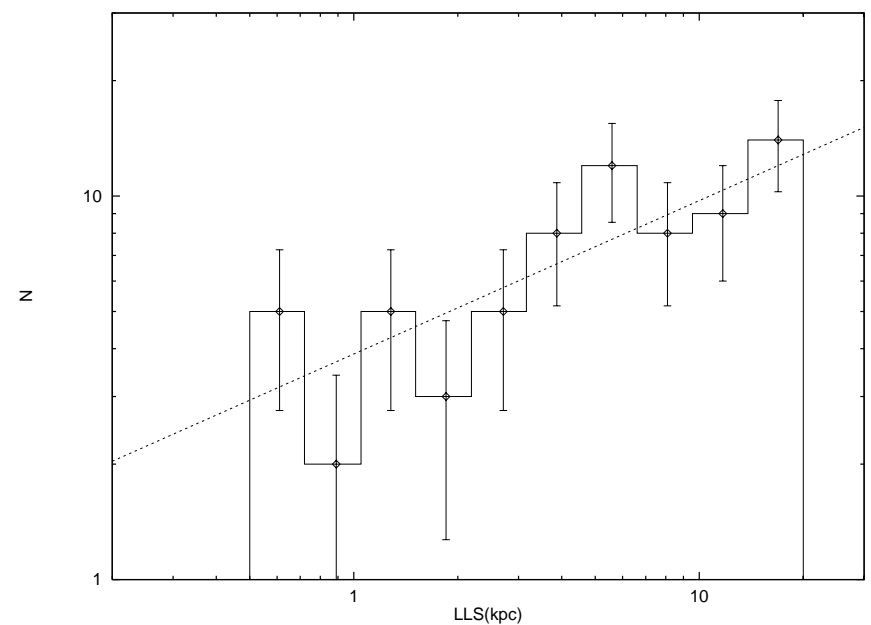

Fig. 9. Distribution function of $L L S$ of the CSSs in the B3VLA sample in bins of $\log ($ size $)=0.16$. The error bars are $\sqrt{N}$. The line is not the best-fit, but it has the slope of 0.4 derived by Fanti et al. (1995) for the $3 C R \& P W$ sample

limit of our selection) down to $\approx 0.4-0.6 h^{-1} \mathrm{kpc}$, corresponding to the resolution limit. However, in many cases, the VLA observations alone allow to define satisfactorily the source morphology only down to $L L S \approx 5 h^{-1} \mathrm{kpc}$, i.e. for $\lesssim 50 \%$ of the whole CSS sample. The remaining objects contain substructures which require higher resolutions for a proper morphological classification. These have already been observed with either the VLBA or the EVN+MERLIN at $1.66 \mathrm{GHz}$ and the results will be presented in forthcoming papers. The $L L S$ distribution can be fitted by the power law $\mathrm{d} N / \mathrm{d}(L L S) \propto L L S^{-0.6}$.

d) The spectra, computed in the range $0.4-8.5 \mathrm{GHz}$, show a significant steepening at high frequencies in the largest majority of cases, as expected from synchrotron electron ageing. A marked flattening at low frequencies is evident for a large fraction of the radio sources with $L L S \leq$ $0.5 h^{-1} \mathrm{kpc}$.

e) The majority of source components are polarized, with median values of their percentage polarization $\approx 6 \%$ and $\approx 4 \%$ at 8.5 and $4.9 \mathrm{GHz}$ respectively.

Results and discussion on points c) to e) will be presented in forthcoming papers.

Acknowledgements. F.P. has been partially supported by a predoctoral fellowship from the Consiglio Nazionale delle Ricerche (CNR). The authors also acknowledge the Italian Ministry for University and Scientific Research (MURST) for partial financial support (grant COFIN 99-02-37). The VLA is operated by the U.S. National Radio astronomy Observatory which is operated by Associated Universities, Inc., under cooperative agreement with the National Science Foundation.

\section{References}

Akujor, C. E., \& Garrington, S. T. 1995, A\&AS, 112, 235

Baldwin, J. 1982, in Extra Galactic Radio Sources, IAU Symp. 97, ed. D. S. Heeschen, \& C. M. Wade (Reidel), 21 
Becker, R. H., White, R., \& Helfand, D. J. 1995, ApJ, 450, 559

Begelman, M. C. 1996, in CygA: Study of a Radio Galaxy, ed. C. Carilli, \& D. Harris (Cambridge University Press), 209

Blundell, K. M., Rawlings, S., \& Willot, C. J. 1999, AJ, 117, 677

Condon, J. J., Cotton, W. D., Greisen, E. W., et al. 1998, AJ, 115,1693

Falco, E. E., Kochanek, C. S., \& Muñoz, J. A. 1998, ApJ, 494, 47

Fanti, C., Fanti, R., Dallacasa, D., et al. 1995, A\&A, 302, 317

Fanti, C. 2000, Proceedings of the 5th EVN Symposium, ed. J. Conway, A. Polatidis, \& R. Booth, Onsala Space Observatory, Chalmers Technical University, Gothenburg, Sweden, in press

Ficarra, A., Grueff, G., \& Tomassetti, G. 1985, A\&AS, 59, 255

Garrington, S. T., Conway, R. G., \& Leahy, J. P. 1991, MNRAS, 250, 171

Kaiser, C. R., Dennet-Thorpe, J., \& Alexander, P. 1997, MNRAS, 292, 73

Katgert-Merkelijn, J. K., Robertson, J. G., Windhorst, R. A., \& Katgert, P. 1985, A\&AS, 61, 517

Laing, R. A., Riley, J. M., \& Longair, M. S. 1983, MNRAS, 204,151

Marecki, A., Falcke, H., Niezgoda, J., et al. 1999, A\&AS, 135, 273

Murgia, M., Fanti, C., Fanti, R., et al. 1999, A\&A, 345, 769

O'Dea, C. P., \& Baum, S. A. 1997, AJ, 113, 148

O'Dea, C. P. 1998, PASP, 110, 493

Owsianik, I., \& Conway, J. E. 1998, A\&A, 337, 690

Owsianik, I., Conway, J. E., \& Polatidis, A. G. 1998, A\&A, $336 \mathrm{~L}, 370$
Patnaik, A. R., Browne, I. W. A., Wilkinson, P. N., et al. 1992, MNRAS, 254, 655

Pearson, T. J., \& Readhead, A. C. S. 1988, ApJ, 328, 114

Peacock, J. A., \& Wall, J. V. 1982, MNRAS, 198, 843

Polatidis, A., Wilkinson, P. N., Xu, W., et al. 1999, 4th EVN/JIVE Symposium, Dwingeloo 22-24 October 1998, ed. M. A. Garrett, R. M. Campbell, \& L. I. Gurvits, New Astron. Rev., 43, 657

Readhead, A. C. S., Taylor, G. B., Xu, W., et al. 1996, ApJ, 460,634

Snellen, I. A. G., Schilizzi, R. T., de Bruyn, A. G., et al. 1998, A\&AS, 131, 435

Snellen, I. A. G., Zhang, M., Schilizzi, R. T., et al. 1995, A\&A, 300,359

Simmons, J. F. L., \& Stewart, B. G. 1985, A\&A, 142, 100

Stanghellini, C., O'Dea, C. P., Dallacasa, D., et al. 1998, A\&AS, 131, 303

Taylor, G. B., Marr, J. M., Pearson, T. J., et al. 2000, ApJ, 541,112

Thompson, D., Djorgoski, S., Vigotti, M., \& Grueff, G. 1994, AJ, 108, 828

Tschager, W., Schilizzi, R. T., Rottgering, H. J. A., et al. 2000, A\&A, 360, 887

van Breugel, W., Miley, G., \& Heckman, T. 1989, AJ, 89, 5

Vigotti, M., Grueff, G., Perley, R., et al. 1989, AJ, 98, 419

Vigotti, M., Merighi, R., Vettolani, G., et al. 1990, A\&AS, 83, 205

Vigotti, M., Gregorini, L., Klein, U., \& Mack, K. H. 1999, A\&AS, 139, 359

Vinokur, M. 1965, Ann. Ap., 28, 412 